\title{
Tropical and Subtropical Cloud Systems
}

\author{
GILLES BELLON AND SANDRINE BONY
}

Tropical and subtropical clouds are characterized by a wide variety of spatial organizations from the mesoscale to the planetary scale. Mesoscale convective systems (MCS) regroup different types of convective and high stratiform clouds and last up to a few days; at the synoptic scale, tropical cyclones (TC) form circular cloud structures with a lifetime of one to two weeks. At larger scales, more cloud systems occur in some regions than others, forming cloud system envelopes that include numerous cloud systems and clear-sky regions in between. Some of these envelopes propagate, such as the convectively-coupled equatorial gravity waves and the Madden-Julian Oscillation (MJO), with lifecycles that last from a couple of days to a couple of months. Others, such as the tropical convergence zones (CZ), are more stationary, but they are still modulated on seasonal timescales, even significantly displaced in the cases of monsoons, and on interannual scales.

Tropical regions differ from higher latitudes by the smallness of the Coriolis parameter. Rotation is therefore relatively unimportant for the tropical circulations, and as a result gravity waves easily disperse temperature anomalies and minimize horizontal temperature gradients in the free troposphere. This means in turn that the energetic balance of the free troposphere is essentially between vertical transport and diabatic terms. This balance is characteristic of the tropics, similarly to the geostrophic balance in the extratropics; it implies a strong interaction between divergent circulation and diabatic heating. Because the tropics absorb a larger amount of solar radiation than the higher latitudes, these diabatic terms are very significant. In this context, clouds are a very active part of the diabatism in the tropics. First, most of the solar energy is absorbed at the surface and a large fraction of it is transmitted to the atmosphere through surface turbulent heat fluxes that warm and moisten the boundary layer; convective clouds redistribute this energy throughout the troposphere by extracting humidity from the boundary layer and carrying it upwards where the latent heat is released by condensation and freezing. For most convective clouds (e.g., congestus and cumulonimbus clouds), this is their dominant diabatic contribution. Second, clouds interact with radiation in both the solar and terrestrial ranges. Some of this radiative effect can be local, cooling or warming the atmosphere, and some of this radiative effect acts remotely by changing radiative fluxes and in particular modulating the surface radiative budget. For some clouds such as cirrus and stratocumulus clouds, their radiative effect is their main diabatic contribution. Rather than causing a uni- form heating, as would be expected from a random spatial and temporal occurrence of clouds, clouds create gradients of diabatic heating and power circulations on many spatial scales because of their multi-scale spatial organization.

On the other hand, cloud occurrence and type are sensitive to the circulation, in particular to the vertical motion of the atmosphere, and to the surface conditions. The twoway interactions between dynamics and clouds and between the surface and clouds underlie feedback mechanisms that are at the heart of many modes of variability of the tropical climate, and some of these feedbacks play an active role in the spatial organization of the atmosphere. However, many aspects of these interactions remain poorly understood.

This chapter aims at presenting the tropical and subtropical cloud systems and their main modes of spatial organization and variability in the light of their interactions with the large-scale tropical circulations and with the surface.

\subsection{Introduction}

The tropics exhibit a large diversity of clouds, from stratiform thin clouds to deep convective clouds. Cloud types can be classified according to their cloud-top pressure and cloud optical thickness (see Chapter 8). Figure 9.1 shows the bi-dimensional distribution of the frequency of cloud occurrence as a function of these properties. High and thin clouds known as cirrus clouds ( $\mathrm{Ci}$ ) are ubiquitous in the tropics, generated by local stratiform condensation or by dissipating convective systems. Low clouds such as the optically-thick stratocumuli (Sc) and optically-thinner, shallow-convective cumuli $(\mathrm{Cu})$ are frequent as well. Stratocumuli populate the eastern subtropical basins while cumuli are ubiquitous in the subtropical trade-wind regions as well as the deep tropics. In convective regions, the cumuli cohabit with deeper congestus clouds $(\mathrm{Cg})$ as well as deep convective clouds (DCC) and their extension of stratiform precipitating clouds (SPC) and non-precipitating anvils (An). In these regions, the distribution of convective cloud-top altitudes is actually trimodal: there is an absolute maximum of shallow cumuli (visible on Figure 9.1 and two secondary maxima for the cumulus congestus and for the deep convective clouds and associated stratiform anvils (DCC, SPC, and An). These three categories of clouds extend approximately from the lifting condensation level up to one of three characteristic layers with enhanced stability. The cumuli reach their maximum heights 
at the trade-wind inversion, a strongly stratified layer at the top of the atmospheric boundary layer between 1 and 3 kilometers of altitude. The congestus clouds develop up to the freezing level around $6 \mathrm{~km}$, where the solid-liquid phase change creates a more stable layer, and the deep convective clouds extend all the way to the tropopause (at about 17 $\mathrm{km}$ of altitude).

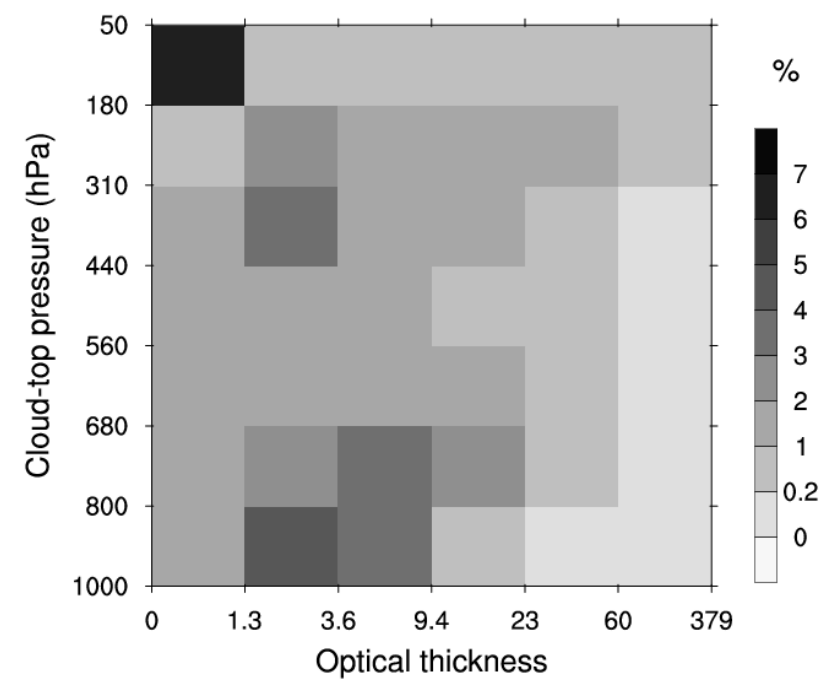

Figure 9.1 Cloud frequency of occurrence stratified in bins of cloud-top pressure and cloud optical thickness (ISCCP data).

The distribution of tropical clouds is rarely random. On the contrary, it is often associated with different forms of organization of the atmosphere over a wide range of scales. For example, stratocumuli form decks over the cool subtropical waters off the western coast of continents, while convective clouds organize in systems from the mesoscale to the planetary scale that are illustrated in Figure 9.2 Mesoscale Convective Systems (MCS) ${ }^{1}$ of tens to hundreds of kilometers such as squall lines, cloud clusters and superclusters, feature a transition from shallow to deep convection, to stratiform rain, as illustrated in Figure 9.3 with a lifecycle of up to a few days. At the synoptic-scale (hundreds to a thousand kilometers), tropical cyclones form circles of convective and high stratiform clouds that last up to two weeks and their extreme versions, the hurricanes, organize deep convection and circulation in spiralling structures around their circular eyewall. At larger scales, these cloud systems can be embedded in envelopes of high cloud activity with other regions witnessing fewer cloud systems. These envelopes are often associated to propagating modes of variability such as the convectively-coupled equatorial gravity waves (on time scales from the synoptic to the intraseasonal) and the 30to-60-day Madden-Julian Oscillation (MJO), which is the most prominent large-scale intraseasonal mode of tropical

\footnotetext{
1 Here we use a wide definition of the Mesoscale Convective Systems regrouping all types of mesoscale convective organization. This term is often used with a narrower definition in the literature.
}

variability. At the planetary scale (thousands to tens of thousands kilometers), convection is spatially organized in Convergence Zones (CZ), where the column-integrated divergence of humidity is negative. These CZs are quasi-steady at the seasonal time scale (see Figure 9.12 or the Atlas for illustrations). The best-known CZ may be the Intertropical Convergence Zone (ITCZ), a rainband that extends zonally along the equator over most of the globe. Monsoons can be seen as a latitudinal shift of the ITCZ (in West Africa), a strengthening of a CZ (the South Pacific CZ or SPCZ, over Australia and the southwestern tropical Pacific), or the emergence of an additional CZ (the Monsoon Convergence Zone around $20^{\circ} \mathrm{N}$ in South Asia).

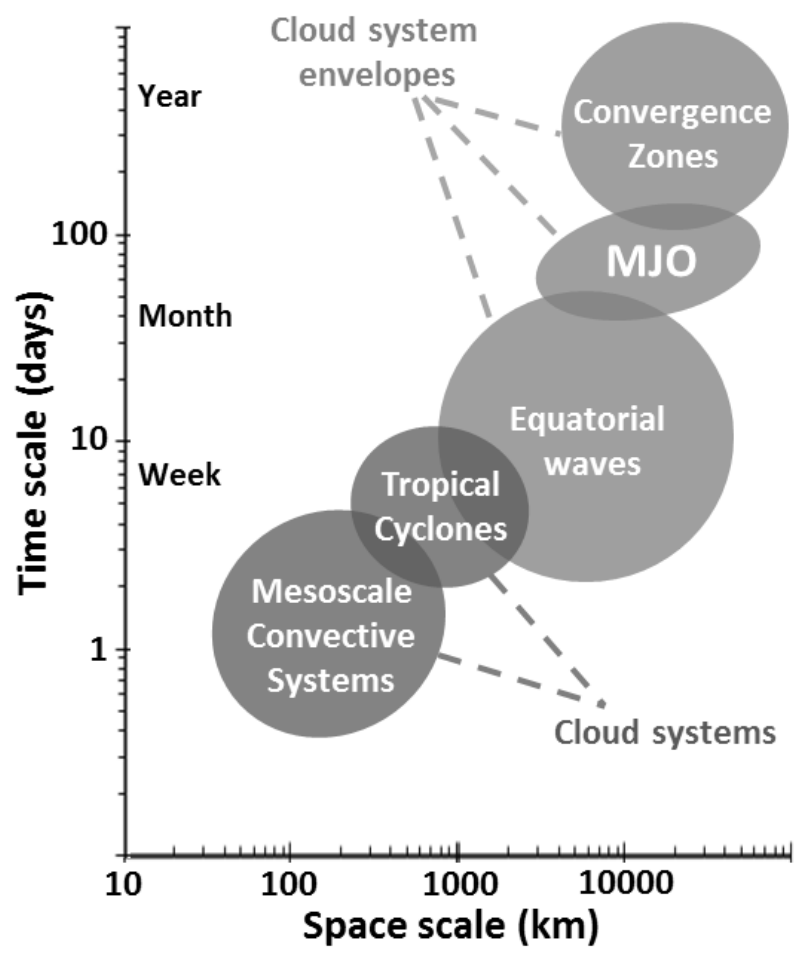

Figure 9.2 Types and scales of organization of tropical convective clouds.

If some of the basic mechanisms of these cloud systems are well understood, there is still a lot of uncertainty on what controls the lifecycle and propagation of MCSs and tropical cyclones, and what determines the location of the CZs, in particular in the case of monsoons. These characteristics result from the interaction of these cloud systems and envelopes with the larger-scale environment, and in particular through interactions with the circulation and the surface.

The spatial organization of clouds is often associated with dynamical patterns that can favor the development of the clouds and/or can be a response to the heating inside the clouds. This association is observed at all scales. At the mesoscale, MCSs can be embedded in easterly waves which propagate westward along monsoon troughs (particularly in Africa) and the ITCZ. An MCS can also create in its wake a mid-level mesoscale vortex that can develop into a severe 
Schematic of a Mesoscale Convective System (MCS)

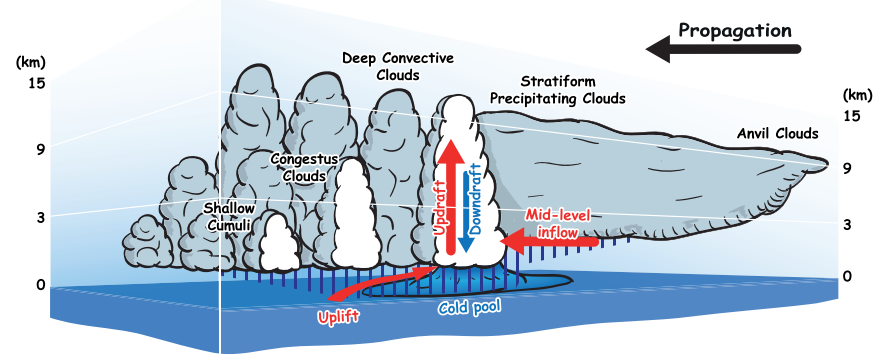

Figure 9.3 Schematic of an MCS.

tropical storm or hurricane, which are associated with strong cyclonic circulations in their mature phase. At larger scale, transient organized convection is often associated with planetary waves. The MJO also exhibits a characteristic dynamical pattern known as Gill circulation which is the equilibrium dynamical response to a steady mid-tropospheric equatorial heating ${ }^{2}$. On the planetary scale, CZs are regions of large-scale convergence in the boundary layer, ascent in the mid-troposphere, and divergence in the upper troposphere. For example, the ITCZ is embedded in the ascending branch of the Hadley circulation, and the rising branches of the Walker circulation are collocated with CZs.

Clouds are coupled with the dynamics through a two-way interaction that is multi-faceted, not perfectly understood, and poorly represented in climate models. On the one hand, the advection associated with a given circulation can influence the temperature and humidity stratifications in an atmospheric column, conditioning the type of mixing and convection that occurs in this column and therefore the type of cloudiness. In particular, vertical transport is crucial, and the vertical velocity exerts a strong control on deep convection, promoting low clouds in the subsiding regions and deep, high clouds in the ascending regions. Horizontal transport can also play a very significant role by carrying away the free-tropospheric moisture, for example in the case of strong vertical shear. On the other hand, clouds can influence dynamics through diabatic heating that changes the temperature stratification and hence the pressure gradients. Most of this diabatic heating is latent, due to the condensation and freezing during the cloud formation and development, or melting and re-evaporation of cloud or precipitating hydrometeors. The clouds also modify the radiative properties of the atmosphere, modulating both the solar and terrestrial radiative heating.

Clouds also exert radiative effects on the surface, and this yields coupled surface-atmosphere interactions. Clouds shield the surface from incoming solar radiation, and the resulting surface cooling decreases the turbulent heat fluxes. The decrease in evaporation dries the boundary layer and this reduces most types of cloudiness (from Sc to DCC). But the decrease in sensible heat flux cools the boundary layer and increases the lower tropospheric stability, a condi-

2 See Exercise 4 on the Gill circulation tion that is unfavorable for convective clouds but favorable for stratocumuli. Over continents, competing mechanisms resulting from the characteristics of the land surfaces give rise to complex feedbacks. This cloud radiative effect on the surface can also influence the circulation through changes in surface air temperature gradients resulting from changes in surface sensible heat flux.

In this chapter, we will illustrate how many features of the tropical climate and its variability result from mechanisms involving clouds. We will first present how clouds can organize spatially either spontaneously or forced by the solar insolation or land-sea contrasts. Second, we will present how clouds interact with the atmospheric circulation, and what mechanisms result from this interaction. Third, we will focus on how the clouds interact with the surface.

\subsection{Clouds spatial organization}

Most of the large-scale features of the tropical cloud distribution (Sc decks, CZs) and their variability (monsoons, ENSO) result from a forcing external to the atmosphere (such as the solar forcing and its seasonal cycle) from the surface heterogeneities (land-sea contrasts, sea surface temperature gradients), or from coupled ocean-atmosphere mechanisms. But spontaneous spatial organization or self-organization, defined as resulting exclusively from atmospheric processes, can be observed frequently as well. At small scales, spontaneous organization can be observed in open and closed cells in stratocumulus decks and the actiniform clouds. At the mesoscale, organized cloud systems are routinely observed in CZs. At the synoptic scale, tropical storms and hurricanes are self-maintaining organized cyclonic systems. At large scales, convectively-coupled equatorial gravity waves and MJO events are forms of spontaneous organization of clouds.

\subsubsection{Mesoscale convective organization}

The cloud clusters and superclusters are of particular importance in the tropical climate variability because most propagating larger-scale tropical modes of variability present a similar cloud structure. These MCSs exhibit a transition from convectively-suppressed conditions to shallow convection, then to deep convection and to stratiform precipitation before a return to dry conditions. This transition in convective regimes corresponds to a transition from cumulus to congestus, to deep-convective clouds, to stratiform precipitation and cirriform anvils, as illustrated in Figure 9.3 . The lifecycle of an MCS lasts from a few hours to a few days. First documented in the tropical Atlantic during the GATE campaign (Global Atmospheric Research Program Atlantic Tropical Experiment) in 1974, tropical MCSs were further documented in the Western Pacific during TOGACOARE (Tropical Ocean Global Atmosphere Program Coupled Ocean-Atmosphere Response Experiment) in 19921993. Recently, satellites such as the Tropical Rainfall Mea- 
surement Mission (TRMM) provide a lot of information of the clouds and precipitation in MCSs.

The transition from shallow to deep convection to stratiform anvil is accompanied by complex cloud-scale circulations. The main features of this circulation are the deep convective updrafts and downdrafts represented in Figure 9.3. The updrafts are composed of positively-buoyant parcels from the boundary layer; upward throughout the deep convective cloud, the moisture in these parcels condenses and rains out, resulting in an enhancement of the parcels' buoyancy. The downdrafts include liquid-water-laden, negativelybuoyant parcels that are cooled on their way down by rain re-evaporation. These downdrafts create a thin layer of cool air at the surface under the deep convective clouds, called a cold pool. Extending around the deep cloud, the cold pool lifts the preexisting, moist and warm surface layer, facilitating the onset of convection ahead of the system. Cold pools also increase the spatial variability of low-level moisture at the MCS scale, and further favor convection around the cold pools. Smaller cold pools occur under shallower precipitating clouds $(\mathrm{Cg}$ and the deepest $\mathrm{Cu})$, and they are active in the transition to deep convection. The MCS is also powered by mid-level inflow from the back of the system. This inflow responds to the pressure gradients created by heating in the deep-convective core and the evaporative cooling by stratiform precipitation.

\subsubsection{Organization at larger scales}

At large scales, multiple modes of variability can be observed in the tropical deep convection. Most of these modes propagate zonally along the equator, and they can be seen in the Hovmöller diagram of deep-convective activity in Figure 9.4 A spatial and temporal filter is used to extract the anomalies corresponding to ranges of wavenumber and frequency of particular interest, and these filtered anomalies are represented by contours in Figure 9.4

Green contours correspond to Kelvin waves that propagate eastward at a speed of about $15-20 \mathrm{~m} \mathrm{~s}^{-1}$, and blue contours correspond to equatorial Rossby waves that propagate westward at a speed of 5 to $10 \mathrm{~m} \mathrm{~s}^{-1}$. Kelvin and equatorial Rossby waves are known solutions of the momentum and dry energy equations of the tropical atmosphere (waves are solutions to the linearized equations). Analytic expressions of these waves' structure and characteristics can be derived from the shallow-water equations on an equatorial beta plane. These equations that describe the homogeneous momentum and depth of an incompressible fluid can also describe the first-baroclinic circulation of the dry equatorial atmosphere. The dynamical structure of Kelvin waves is non-rotational, symmetric with respect to the equator, with alternating regions of ascent and descent along the equator resulting from the convergence or divergence of the zonal wind. The Rossby waves feature alternating pairs of low-level cyclonic gyres and pairs of anticyclonic gyres straddling the equator. Another prominent observed type of waves, the Mixed Rossby-Gravity waves, is not visible in Figure 9.4 because its signal is antisymmetric about the equator. These equatorial waves (Kelvin, equatorial Rossby, and

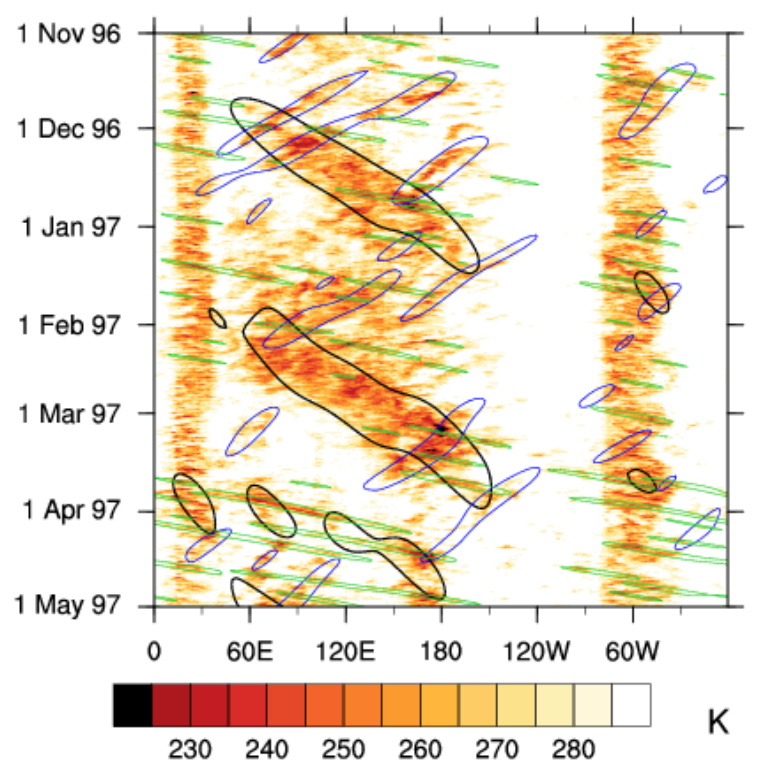

Figure 9.4 Example of a Hovmöller diagram of the equatorial brightness temperature anomalies (shading) and filtered anomalies (contours) for the Kelvin waves (green contours), equatorial Rossby waves (blue contours), and MJO (black). Low brightness temperatures correspond to deep clouds. Data from the Cloud Archive User Service (CLAUS).

Mixed Rossby-Gravity waves) are observed in the tropical atmosphere. They can be associated with deep convection; they are then called convectively-coupled equatorial gravity waves and propagates more slowly than the dry waves. The influence of moist physics on the wave dynamics can be accounted for by a simple change of parameter to take into account the impact of moist convection on the mean thermal structure of the atmosphere. The propagation speed is reduced because of this parameter, but the wave structure is not significantly modified by this coupling. This means that moist processes do not play an active part in determining the structure of these waves, although clouds feed back on the dynamics and can be instrumental in exciting and sustaining the waves. Despite our theoretical understanding of these waves, many climate models based on the primitive equations are still very poor at simulating them.

The black contours in Figure 9.4 correspond to the Madden Julian Oscillation (MJO). This large-scale convective disturbance propagates eastward along the equator from the Indian Ocean to the Central Pacific with a propagation speed of about $5 \mathrm{~m} \mathrm{~s}^{-1}$. The MJO has a characteristic dynamical pattern with a pair of off-equatorial, low-level cyclonic gyres straddling the equator west of the convective disturbance, a strong surface westerly jet between these gyres, surface easterlies over an extended region east of the disturbance, and the opposite circulation in the upper troposphere. There is mid-tropospheric ascent around the convec- 
tive region and subsidence elsewhere. This pattern is similar to a Gill circulation 3 in equilibrium with the latent heating from the MJO convection. The dynamical signal regularly propagates around the globe from the East Pacific back to the Western Indian Ocean with a faster propagation speed of about $20 \mathrm{~m} \mathrm{~s}^{-1}$, resulting in a period of about 40 days. This extension of the dynamical propagation might involve a conversion of the disturbance into a dry Kelvin wave.

First documented in the 1970s, the MJO still eludes our theoretical understanding. It is thought to be a moisture mode, i.e., moisture, and in particular moisture transport, is crucial to its development (and it cannot be described by the equations for a dry atmosphere). But no simple model of the MJO has been widely accepted yet. The fundamental mechanism of the MJO results from the interaction between clouds and circulation, but it is not known which aspects are essential, and ocean-atmosphere coupling is thought to be important as well.

Simulation of the MJO by general circulation models has been improving, but it is still poor in most climate models. At the large scale, these models are expected to represent accurately the dynamics, so the failure to simulate the MJO is attributed to the parameterizations of moist physics (and to the feedbacks between dynamics and moist physics). Two flaws of these parameterizations are hypothesized to play a role in this failure:

- The parameterizations do not produce the correct profile of diabatic heating associated with the MJO. This yields biased pressure gradients and the resulting dynamical response does not provide the appropriate transports of moisture and energy necessary for the development and propagation of the MJO.

- The parameterizations are unable to simulate the spontaneous, upscale organization of convection from the subgrid scale to the large scale, which prevents the development of a large-scale dynamical response which would then be able to further develop and propagate the MJO disturbance.

In particular, parameterizations of deep convection are suspected to underestimate the sensitivity of convection to free-tropospheric humidity, a factor that could underly both flaws.

As mentioned above, the large-scale modes of atmospheric variability such as the convectively-coupled equatorial gravity waves or the MJO have a cloud signature similar to the MCSs, even though their spatial scales are much larger and their temporal scales are better measured in days or weeks rather than in hours. Figure 9.5 shows the composite cloud signatures of the MJO and Kelvin wave. These cloud patterns are indeed similar and exhibit a transition from shallow cumuli to deep-convective clouds to stratiform anvils similarly to MCSs.

The convectively-active, ascending phases (phases 4 and 5) of the MJO and Kelvin wave are associated with maxima in all types of clouds $(\mathrm{Cu}, \mathrm{Cg}, \mathrm{DCC}, \mathrm{SPC}, \mathrm{An})$. Shallow convection starts deepening in the subsiding free troposphere two to three phases beforehand, and the occurrence of congestus increases. At the same time, cloudiness starts increas-

3 See Exercise 4 for details. ing at the tropopause thanks to very intense, isolated deep convective clouds. Organized deep-convective and stratiform precipitating clouds develop one to two phases before the MJO peak, and remain for one phase afterwards, with lowering cloud tops. Anvil clouds linger for another phase afterwards during phases of subsidence.
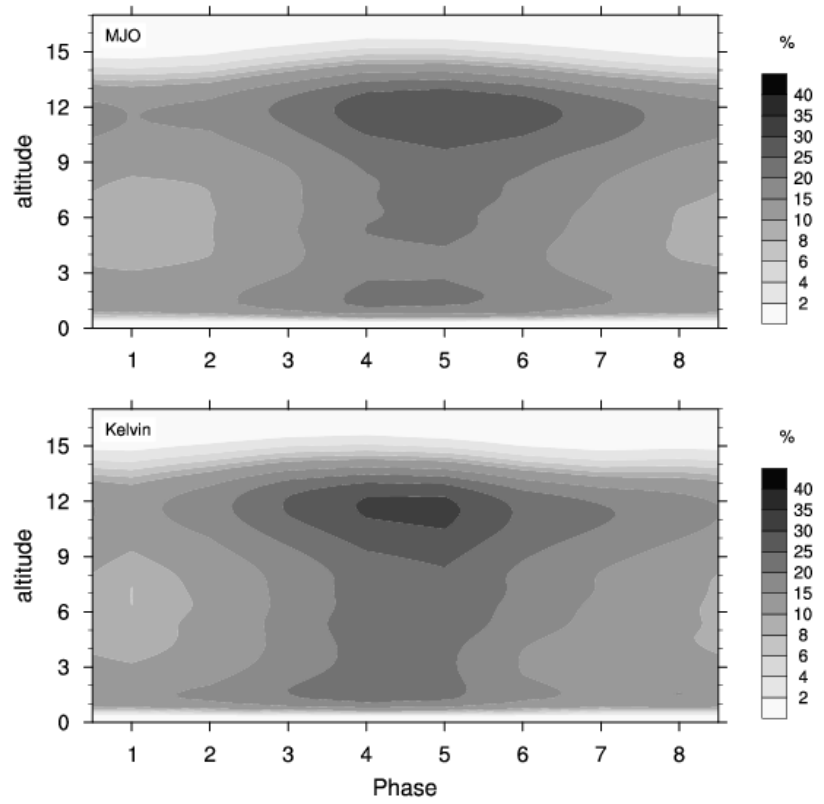

Figure 9.5 Cloudiness as a function of the phase of the Madden-Julian Oscillation and convectively-coupled Kelvin wave (CloudSat, $15^{\circ} \mathrm{S}-15^{\circ} \mathrm{N}$ ). The $\mathrm{x}$-axis can be considered as a time axis, with a typical timescale between two phases of about 5 days for the MJO and 1 day for the Kelvin wave.

This similarity of the cloud patterns of MCSs and largerscale modes of variability (such as Kelvin waves and MJO) is quite intriguing, especially since the large-scale envelopes of MJO events or Kelvin waves often contain full lifecycles of MCSs (as can be seen in Figure 9.4 e.g., around $60^{\circ} \mathrm{E}$ on Nov. $\left.22^{\text {nd }}, 1997\right)$. This raises the question whether cloud variability follows a self-similarity across scales due to some fundamental cloud mechanism.

The "stretched building block" hypothesis aims at explaining this similarity, suggesting that depending on the phase of the large-scale wave, the MCS lifecycles (which are the building blocks) are modified, with one phase of these lifecycles lasting longer or covering more surface (stretched) than in an average lifecycle. From this viewpoint, MCSs during the pre-convective phase of the large-scale wave have a longer or larger than average shallow-convective phase in their lifecycle, while MCSs during the mature phase have a larger than average deep convective cloud fraction, and MCSs during the decaying phase exhibit a larger stratiform anvil. These variations in the duration or size of the different phases of the MCS lifecycle are likely to result from their sensitivity to the large-scale environment that is modulated by the large-scale modes. In particular, the sensitivity to the large-scale circulation is expected to play a major role: convergence of the horizontal wind favors deep convection, 
ascent can sustain a stratiform anvil, and subsidence damps deep convection, limiting convection activity to the boundary layer. Still, this hypothesis has not been validated. How the internal mechanisms of MCSs (updraft, cold-pool uplift) react to larger-scale variability, and how MCSs give rise to, or feed back onto, larger-scale variability has yet to be understood.

\subsubsection{Meridional organization due to insolation}

The solar heating of the atmosphere and surface induces cloud spatial structures at the planetary scale and, despite its weak annual cycle in the tropics, forces a very significant seasonal variability.

The average insolation is maximum at the equator. As a result, the sun heats the equatorial atmosphere more than the subtropical atmosphere. Part of this meridional gradient of heating is due to direct atmospheric absorption of solar radiation, but most of it is actually mediated by the surface, through terrestrial radiation and turbulent heat fluxes. Indeed, the sea surface temperature (SST) is warmer at the equator (or nearby if there is upwelling of deep waters at the equator) than in the subtropics. In response to this heating gradient, a meridional circulation arises with ascent around the equator, poleward motion in the upper troposphere, subsidence in the subtropics and equatorward motion in the lower troposphere. It is known as the Hadley circulation. The Hadley circulation can be modeled by very simple equations of a dry atmosphere, such as the shallow-water equations on an equatorial beta plane. Simple models predict the near-surface easterly trade winds and the subtropical upper-tropospheric jets based on angular momentum conservation, though it has been shown that eddies due to the instability of these jets limit the applicability of angular momentum conservation. Indeed, moist processes are essential to the real-world Hadley circulation, since they control how the surface heating gradients are transmitted to the free troposphere (or not) and since they create a feedback between circulation and heating.

The cloud patterns embedded in the Hadley circulation are on the planetary scale, clearly visible from space. Figure 9.6 shows a typical latitude-altitude view of the Hadley circulation with the associated cloud patterns 4 here for the boreal summer in the Atlantic Ocean. In the subtropical descending branch of the Hadley circulation, deep clouds are scarce, boundary-layer clouds are ubiquitous over the oceans, and continents tend to be desertic. Shallow cumuli are abundant in most of the subtropical oceans except for the eastern coastal regions where the sea surface temperature is

\footnotetext{
4 Note that in the GOCCP data used here and in the following figures, a systematic minimum on the vertical profile of cloudiness in convective regions can be seen between 3 and $5 \mathrm{~km}$ of altitude. This feature might be more prominent in the GOCCP data than in the real world because this dataset is based on lidar observations from the satellite Calipso, and it is difficult for space lidars to detect clouds overlapped by other thick clouds. But this minimum has also been noted in satellite infrared observations and surface observations. It results primarily from the fact that this layer of the troposphere is less stable than the inversion below and the layer around the freezing level above.
}

cool and stratocumuli form decks. The shallow convection deepens equatorward and congestus clouds appear. In the ascending branch of the Hadley circulation lies the ITCZ, that extends along the equator (between 5 and $10^{\circ} \mathrm{N}$ over most of the tropical oceans). The ITCZ is populated by all types of convective and high clouds $(\mathrm{Cu}, \mathrm{Cg}, \mathrm{DCC}, \mathrm{SPC}$, An).

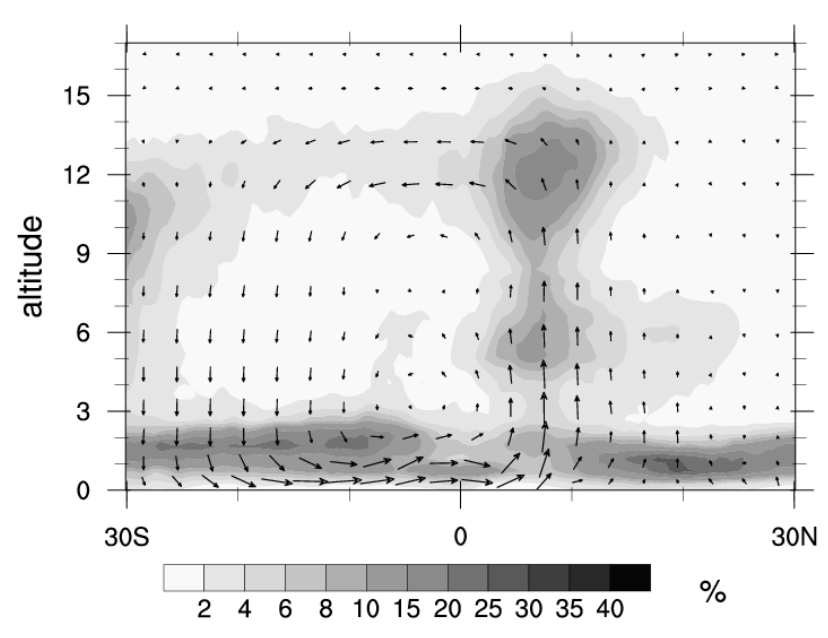

Figure 9.6 Average boreal-summer cloudiness (shadings, GOCCP) and circulation (vectors, ERA-Interim) over the Atlantic ocean $\left(40^{\circ} \mathrm{W}-20^{\circ} \mathrm{W}\right)$.

Because the insolation does not vary seasonally as much in the tropics as in the extratropics, the seasonal cycle in the tropical belt is generally small over the ocean. But in regions with a subtropical continent, the interaction between ocean, land, and atmosphere creates the most prominent features of the seasonal cycle in the tropics: the monsoons. Depending on the criteria for the seasonality of precipitation and surface winds used to define a monsoon, three 5 or five monsoons can be observed on Earth: the Asian and West African monsoons (in boreal summer) and the Australian monsoon (in austral summer) are the most prominent monsoon systems. The North American and South American monsoons are smaller, less-pronounced monsoon systems that are observed, respectively, over the Southwestern North America in boreal summer and over Amazonia in austral summer.

Figure 9.7 shows the cloudiness in the West African sector in winter and summer. The West African monsoon is characterized by a northward shift of the ITCZ from its winter equatorial location to its summer monsoonal location around $12^{\circ} \mathrm{N}$, accompanied by an enhancement of the ITCZ cloudiness. In the austral tropics, increased subsidence

5 Using Ramage's criteria:

- Prevailing wind direction shifts by at least $120^{\circ}$ between January and July.

- Prevailing wind direction persists for at least $40 \%$ of the time in January and July.

- Mean wind exceeds $3 \mathrm{~m} / \mathrm{s}$ in either month.

- Fewer than one cyclone-anticyclone alternation occurs every 2 years in either month in a $5^{\circ}$ latitude-longitude square. 
during the monsoon favors an increase in low-cloud cover. The Hadley circulation intensifies during the monsoon, with an enhancement of the asymmetry between hemispheres, the development of a strong cross-equatorial flow, and a reversal of the trade winds in the summer hemisphere (not shown). This is the canonical pattern of a monsoon, except for the clouds in the middle troposphere over the northern subtropics, that sit atop the Saharan low and are specific to the West African monsoon.
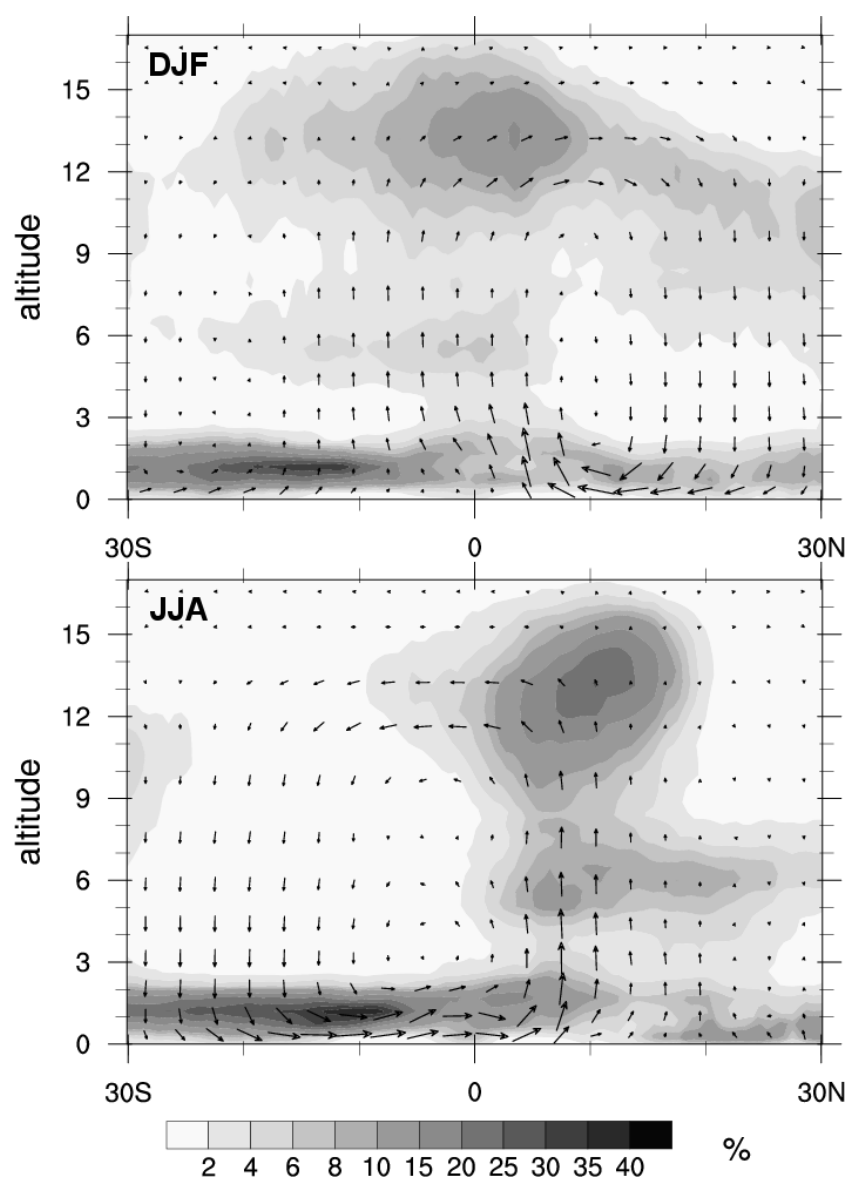

Figure 9.7 Average boreal winter and summer cloudiness (shadings, GOCCP) and circulation (vectors, ERA-Interim) over West Africa $\left(20^{\circ} \mathrm{W}-20^{\circ} \mathrm{E}\right)$.

Other monsoons can exhibit features that differ from this canonical pattern. The Asian monsoon is characterized by two CZs in the Indian sector, one over the equator and one around $20^{\circ} \mathrm{N}$, and the Australian monsoon is associated with an intensification of the SPCZ.

Monsoons can be thought of as the Hadley circulation resulting from off-equatorial warm surface temperatures. These warm temperatures generate large turbulent surface fluxes that create convective instability, favoring the development of convective clouds that in turn anchor an offequatorial CZ over the warm surface. Typically, for the solar annual cycle to produce such warm surface temperatures, the surface heat capacity has to be small, as in the case of land surfaces, so that the surface temperatures adjust very fast to the solar forcing. But in the case of continents, a high surface albedo can reduce the solar warming of the surface and limit the poleward extension of monsoons. This is what happens over subtropical deserts.

Although the Hadley circulation can be understood using simple equations of the dry atmosphere, understanding and simulating the observed circulation and clouds has proved somewhat unsuccessful. There is no consensus understanding of what controls the ITCZ regime: why is the oceanic ITCZ north of the equator? Why are monsoons characterized by distinct ITCZ patterns? This is particularly vexing because the simulation of these features by general circulation models is unsatisfactory and some theoretical understanding would help improve them. These models are still challenged by the simulation of monsoons that are often underestimated. And they tend to simulate two ITCZs straddling the equator rather than one ITCZ north of the equator, a systematic bias known as the "double ITCZ syndrome". Although a double ITCZ can be observed in the eastern Pacific during boreal spring, models tend to simulate it half the year in this region, and some simulate it over the Atlantic as well.

\subsubsection{Zonal organization due to surface heterogeneity}

Figure 9.8 shows the climatological cloudiness and circulation over the Equator $\left(10^{\circ} \mathrm{S}-10^{\circ} \mathrm{N}\right)$. From the Eastern Indian Ocean to the Western Pacific, as well as over Amazonia and Central Africa, ascending motion can be observed over the equator. In between, regions of subsidence can be observed with mean easterlies at the surface, except in the western equatorial Indian oceans where the surface winds are westerlies, converging into the regions of ascent. This circulation is known as the Walker circulation. In the ascending branches of the Walker circulation, all types of convective and precipitating clouds $(\mathrm{Cu}, \mathrm{Cg}, \mathrm{DCC}, \mathrm{SPC}, \mathrm{An})$ occur frequently, while the descending branches are populated by cirrus and low clouds. In these regions of reduced elevated cloudiness at the equator, a well-defined ITCZ is located off the equator (as in Figure 9.6. Figure 9.8 makes it clear that the continents and their orography play a role in the emergence of the Walker circulation, since rising branches are over continents and islands. In particular, the orography blocks the easterly trade winds, which are prevalent in the Pacific and Atlantic Oceans (they are in fact part of the Hadley circulation), and this mechanically forces ascending motions over the Maritime Continent and Amazonia. Other mechanisms such as sea-breeze systems and land-atmosphere feedbacks contribute to the locking of CZs over continents and islands. In the equatorial Pacific, and to some extent in the Atlantic as well, the trade winds also force upwelling of deep, cold water in the eastern part of the basin and push warm equatorial waters to the western part of the basin, creating an east-west slope of the thermocline. This favors deep convection in the western part, particularly in the equatorial West Pacific CZ (WPCZ).

The main mode of interannual variability in the planetary climate is essentially a variation of this mechanism involving surface temperature and wind. It is the El Niño - 


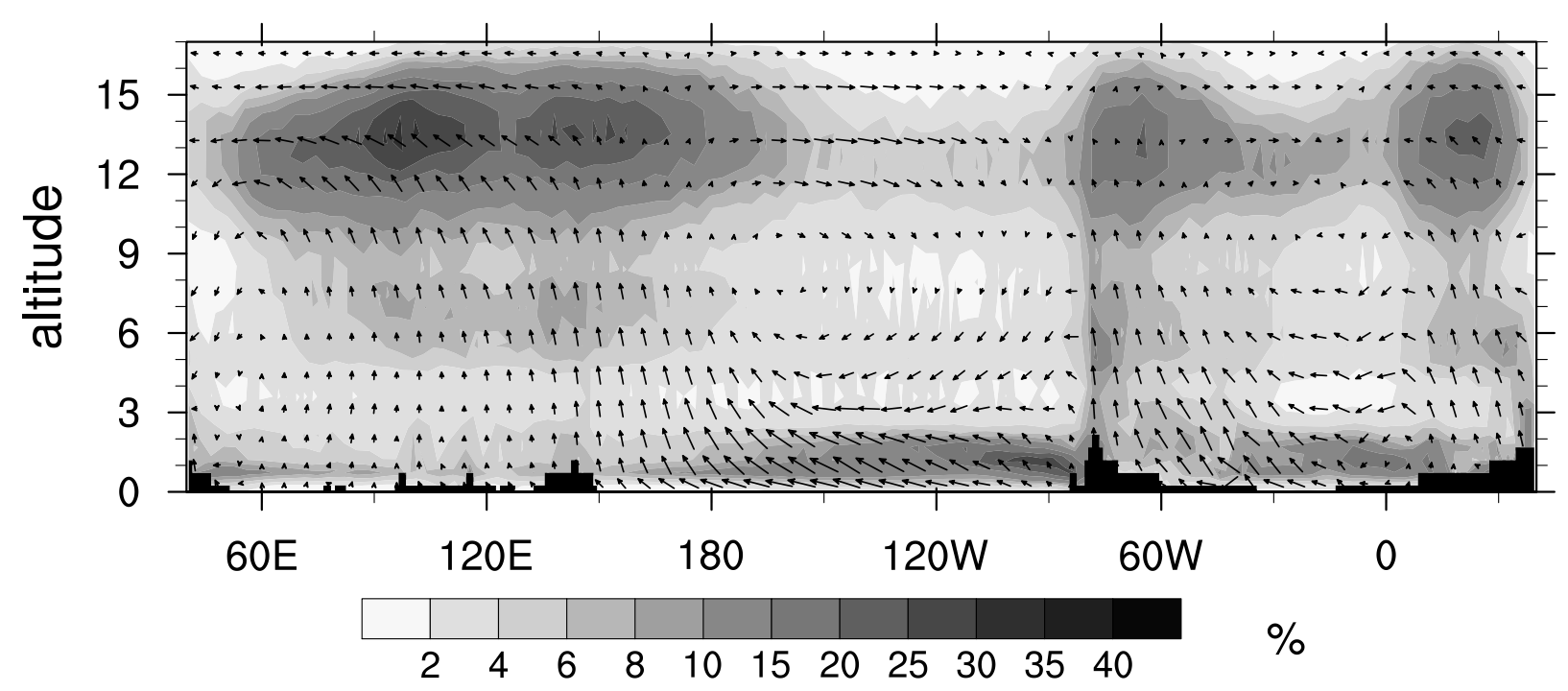

Southern Oscillation (ENSO), whose warm phase, El Niño, is characterized by a warming of the Central and East Pacific equatorial surface waters, collocated with an increase in precipitation and associated with a weakening of the equatorial easterly winds and a flattening of the thermocline. The cold phase, La Niña, consists of an enhancement of the east-west sea-surface temperature and precipitation gradients in the equatorial Pacific, with a reinforcement of equatorial easterly winds. El Niño can be seen as an eastward shift and a weakening of the Walker circulation, as well as a strengthening of the Hadley circulation: the Western Pacific CZ moves eastward and east-west contrasts of deep convection and vertical motion are reduced. Conversely, La Niña is associated with an enhancement of the Walker circulation and a weakening of the Hadley circulation.

Figure 9.9 shows the anomalies of cloudiness associated with both phases of ENSO. During El Niño, convection in the WPCZ weakens and shifts eastward, resulting in a marked increase in high and mid-level cloudiness over the equatorial Central Pacific, extending over the equatorward margin of the ITCZ in the East Pacific. Convection and the associated middle and high clouds also intensify in the Western Indian Ocean, and weaken in Amazonia as a result of the shift of the Walker circulation. In the subtropical Pacific, the enhanced subsidence in the descending branch of the Hadley circulation inhibits convection and results in a decrease in middle and high clouds. In general, El Niño lowcloud patterns are opposite to the high cloud patterns in the Pacific Ocean: deep convection replaces shallow convection (and low clouds occurring below high clouds are not detected by the satellites used to produce the ISCCP data) in the Central and East equatorial Pacific, and the opposite change occurring in the subtropical Pacific and over the equatorial Maritime Continent. But low clouds also decrease in the subtropics around the Maritime Continent and in the Eastern Indian Ocean, and increase in the Western Indian Ocean independently from the deep-cloud patterns, probably due to the reorganization of the Hadley/Walker circulation.
Figure 9.8 Climatological cloudiness (shadings, GOCCP) and circulation (vectors, ERA-Interim) over the Equator $\left(10^{\circ} \mathrm{S}-10^{\circ} \mathrm{N}\right)$. Equatorial orography is filled in black.

During La Niña, the cloud anomalies are generally opposite to the El Niño anomalies, but they are smaller and only the main patterns are visible.

\subsubsection{Convective self-aggregation}

Cloud-resolving model simulations run over large domains and general circulation models have shown that under certain conditions, the horizontally homogeneous RadiativeConvective Equilibrium (RCE) could become unstable. This instability manifests itself by the spontaneous tendency of the atmosphere to organize into dry and moist areas associated with large-scale descent and ascent, respectively. The process generally starts with the formation of a dry patch that expands, eventually forcing the convection to aggregate into narrow areas, in the form of a few clusters, at the edges of or in-between the dry patches. This phenomenon is referred to as "convective self-aggregation", and could play a significant role in the development of MCSs. The physical mechanisms responsible for RCE instability and self-aggregation are still a matter of research. However, the role of interactions between atmospheric water (in the form of water vapor and clouds), radiative cooling and circulation, between surface wind anomalies and surface turbulent fluxes, and between moisture and convection have all been shown to play an important role in the initiation and development of self-aggregation. On the other hand, the maintenance of self-aggregation seems to rely primarily on the interaction between deep convective clouds and radiation, and in particular the reduction of the atmospheric radiative cooling by clouds. Both numerical and observational studies show that situations associated with more aggregated convection are generally drier and less cloudy in the upper troposphere, which tends to increase the emission of longwave radiation to space and to reduce the planetary albedo. This 


\section{ISCCP cloudiness anomaly El Nino / La Nina}
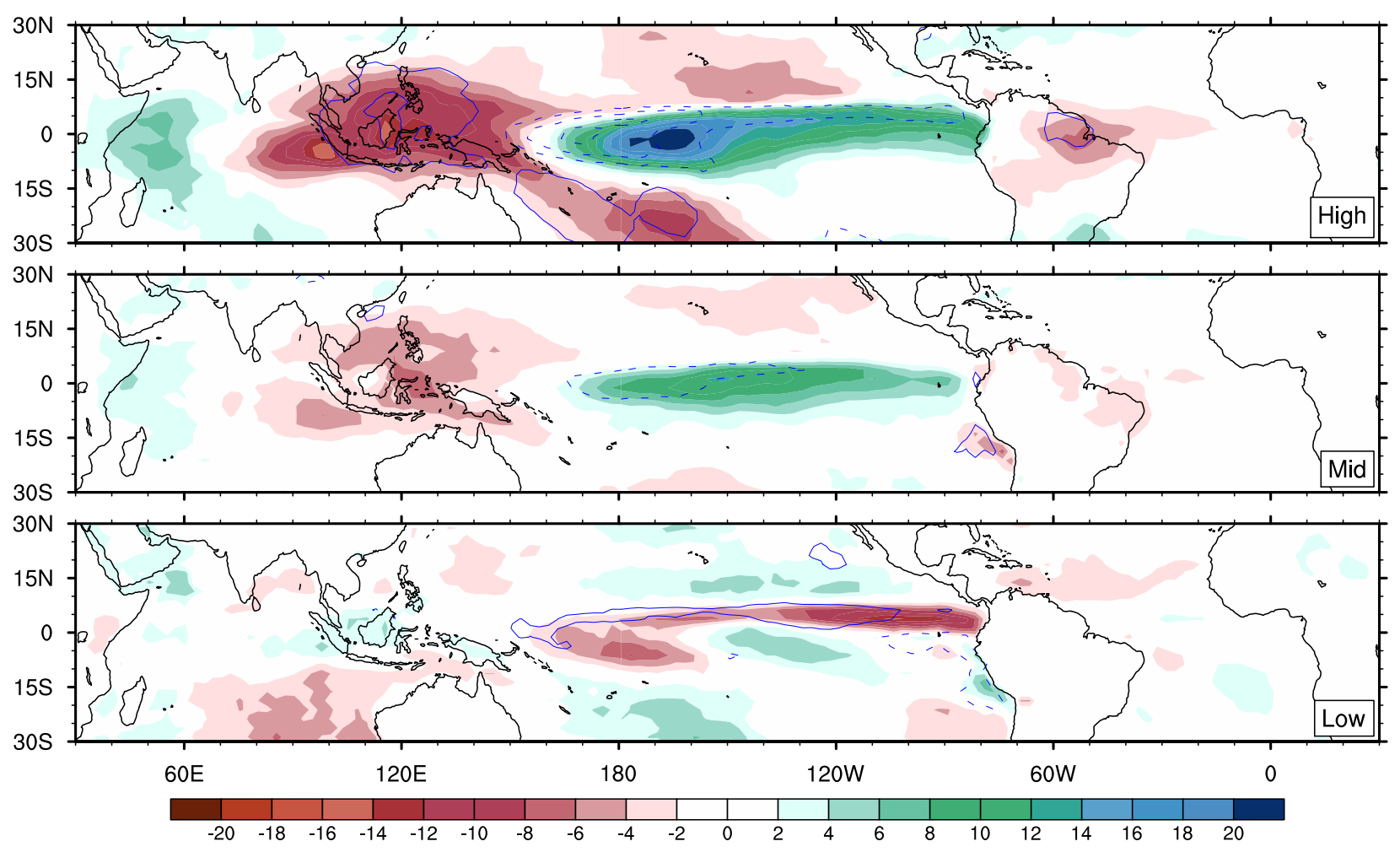

impact suggests that the spatial organization of convection might have a climate impact. Furthermore, models suggest that the phenomenon of self-aggregation is temperature dependent, and that it is favored at high surface temperatures (some studies suggest that it can also be favored at very low temperatures). Given the impact that convective aggregation has on the large-scale atmospheric state and the radiation budget, the question thus arises whether this temperature dependence might affect climate feedbacks (see chapter 13). Although a few studies suggest that the MJO might be a large-scale manifestation of convective self-aggregation, the relationship between this phenomenon and the large-scale organization of convection is still an open question.

\subsection{Cloud-stratification interaction}

We have shown in the previous section that clouds exhibit a vast diversity of organization, on a large range of scales; this has been known since the 1970s thanks to campaign and satellite observations. What is now increasingly recognized is the fact that clouds play an active role in these different forms of organization. It stems from the different ways through which clouds interact with their environment. Clouds are sensitive to the environmental atmospheric stratification, and affect their environment in turn through moistening and heating.
Figure 9.9 High, middle and low cloudiness anomalies (ISCCP) for the different phases of ENSO: El Niño (shading) and La Niña (contours: interval is $4 \%$, solid for positive anomalies, dashed for negative anomalies, and the zero contour is omitted).

Temperature stratification has a strong influence on clouds, because it constrains the static stability of the atmosphere and thus the type of clouds that develop. For large Lower-Tropospheric Stability (LTS $=\theta(700 \mathrm{hPa})$ $\theta$ (surface)), convection is limited to the boundary layer whose depth decreases with increasing LTS. The occurrence of stratocumulus clouds exhibits a strong positive correlation with the LTS. For lower LTS, shallow convection and shallow cumuli occur. Deep convective clouds are associated with low LTS, but in this case it is the convective mixing that controls the LTS by setting the lower-tropospheric temperature profile to a moist adiabat.

Clouds are also sensitive to humidity: boundary layer moisture determines, together with temperature, whether and which boundary-layer clouds develop, and is the reservoir for convective moistening by shallow and deep convection. And environmental free-tropospheric humidity has been increasingly recognized as a key variable for cloud development at all scales. In subsiding regions, low-level clouds are sensitive to lower-tropospheric humidity because it regulates the ventilation of the boundary layer by subsidence and the buoyancy of mixed parcels at the top of the boundary layer. In general, the moister the free troposphere, the larger the low-cloud cover. Convective clouds are also sensitive to the free-tropospheric environmental humidity because of 
the lateral entrainment of environmental air into convective plumes. This mixing dries (and cools) the updrafts, reducing their buoyancy, and dampens or even inhibits deep convection. Regions with a moist free troposphere are therefore favorable for deep convection.

In turn, convective clouds influence the large-scale humidity profile via convective mixing and detrainment, which redistributes boundary-layer humidity within the atmospheric column, and via precipitation, which dries the troposphere. This interaction between clouds and humidity introduces moisture-convection feedbacks that appear to be critical for many phenomena, from convective self-aggregation to the location of the $\mathrm{CZs}$, and in particular in the lifecycle of spontaneously organized convection from the mesoscale to the intraseasonal scale.

Interactions between clouds and the thermodynamical vertical structure of the atmosphere are essential to the life cycle of the MCS. Deep convection is sensitive to freetropospheric humidity because of convective entrainment. Through convective mixing, cumulus and congestus clouds moisten the free troposphere and facilitate the onset of organized deep convection, although this effect might not be as large as the effect of cold pools resulting from congestus convection (see Section 9.2.1). The water detrained from deep convective clouds themselves moistens the free troposphere and this moistening helps sustain deep convection, creating a moisture-convection feedback. On the other hand, deepconvective and stratiform precipitating clouds warm the upper troposphere and cool the lower troposphere. This thermal restratification of the troposphere damps convection; it is instrumental in the demise of the MCS. Precipitating clouds also desiccate the atmospheric column, which damps convection as well. How these different mechanisms compete or cooperate to control the lifecycle of MCSs remains an open area of research.

This combination of effects is also active in larger-scale variability such as in Kelvin waves and the MJO. It contributes to the transition from shallow to deep convection, from convective to stratiform precipitation and anvil clouds observed in such large-scale modes. In particular, lowerfree-tropospheric humidity increases ahead of the convective maximum of the MJO, due to processes that are still debated but moistening by shallow cumulus, congestus and isolated deep convective clouds is thought to contribute.

Understanding and quantifying how the different sensitivities and contributions play out to determine moistureconvection feedbacks is still a matter of research. Representing this interaction between clouds and large-scale environment is essentially the goal of moist-physics parameterizations, and our lack of a complete understanding of these processes is one of the reasons why parameterizing clouds and convection is still challenging. Chapter 7 on parameterizations details these processes and in the following we will focus on indirect effects, due to processes that change the large-scale thermodynamic stratification and that clouds in turn influence. The most important of these is the atmospheric circulation.

\subsection{Cloud-circulation interaction}

In the midlatitudes, where the Coriolis parameter is larger than in the tropics and the potential latent heat release is smaller because of the limited amount of moisture in the atmosphere, Earth's rotation is more important for atmospheric dynamics than diabatic processes, although the latter have recently been found to be very significant. Conversely, the diabatic processes are at the core of tropical circulations, and a large part of these diabatic processes occur within clouds. Heating by clouds creates horizontal gradients of temperature between the cloudy regions and the surrounding clear-sky regions, and the dynamics respond to the resulting pressure gradients. The dynamics in return affects the cloudiness because the associated energy and moisture transport can change the environmental stratification, and hence the types of cloud that will develop or decay.

The interaction between clouds and dynamics is essential in almost every aspect of the tropical climate and its variability. Its most prominent facet is the interaction between deep convection and ascent. Both processes taken independently are essentially self-attenuating (except for dry waves that are free solutions of the momentum and dry energy equations): deep convection is damped by the thermal restratification and boundary-layer drying associated with deep clouds, and ascent of dry air is damped by the pressure gradients created by the advective cooling it causes. But the interaction between these two processes can maintain deep-convective patterns associated with large-scale ascending motion on longer timescales. Historically, as early as the 1960s, the interaction between large-scale circulation and deep convection was thought to be crucial to the atmospheric variability in the tropics. This led to the development of the theory of Convective Instability of the Second Kind (CISK) that describes the growth of all tropical disturbances as a result of a strong positive feedback between low-level convergence caused by mid-tropospheric latent heating and precipitation caused by the convergence of humidity. This theory inspired closures on the column-integrated convergence or moisture convergence in the parameterization of deep convection. It was shown later that this theory and the related closures exaggerate the role of the circulation in controlling convective clouds and can even suggest a wrong causality relationship between moisture and circulation.

In terms of amplitude, latent heat release in convective clouds is the dominant contribution to diabatic heating in the tropical atmosphere. As a result, most tropical modes of variability involve a convective component. By converting latent energy into internal energy, this contribution does not change the energy content of atmospheric columns, but it can create strong gradients of temperature and pressure.

Another important way through which all clouds interact with circulations is through radiation. Deep convective clouds radiatively warm the troposphere while boundarylayer clouds radiatively cool the atmosphere (see Chapter 4). Low-cloud radiative effects strengthen the winds at the surface of tropical oceans and amplify the atmospheric overturning circulation. Deep convective clouds exert an even 
stronger impact on circulations. First they warm the atmosphere, which increases the static stability of the free troposphere, shrinks the anvil cloud amount, and enhances the horizontal gradients of atmospheric radiative cooling between convective and non-convective areas. These effects were found to impact the organization of convection over a large range of scales, and the structure of large-scale convergence zones. Second, they enhance the large-scale meridional gradients in upper-tropospheric temperature, which affects the baroclinicity of the extratropical atmosphere and the mid-latitude jets. Finally, as deep clouds radiatively warm the atmosphere during the rising phase of large-scale tropical disturbances and low clouds radiatively cool the atmosphere during episodes of large-scale subsidence, cloud-radiative effects reduce the effective stratification felt by propagating waves and slows down their propagation. Consistently with these different influences, cloud-radiative effects have been found to influence tropical intraseasonal oscillations, ENSO variability, the intensity and width of the ITCZ, the position and strength of extratropical storm tracks, and the structure and intensity of tropical cyclones.

\subsubsection{Influence of circulation on cloudiness}

Vertical motion can influence the environmental stratification very efficiently. In the free troposphere where humidity decreases with altitude and dry static energy increases, ascent moistens and cools while subsidence dries and warms. Within the boundary layer where the vertical gradients of conserved variables are small, vertical advection is very small. As a result, the lower-tropospheric temperature and humidity stratification are affected by vertical motion. Note that large-scale ascent refers to an average value that conflates updrafts and downdrafts within convective clouds with slow, mostly subsiding motion in the environment (which means that vertical transport is actually more complex than pictured here, although the overall contribution follows the same principles), while large-scale subsidence averages more horizontally uniform values.

By decreasing tropospheric stability and moistening the free troposphere, ascent favors deep convection: the development of all types of middle and high clouds are facilitated by large-scale ascent. Conversely, subsidence warms and dries the free troposphere, thus increasing the LTS and limiting convection and clouds to the boundary layer. Once an inversion forms at the top of the boundary layer, subsidence and cloud-top entrainment also transport warm and dry air into the boundary layer, a process known as ventilation which tends to reduce cloudiness there. But a large LTS also keeps the boundary layer shallow and moist, favorable to stratocumulus formation characterized by a large cloud cover.

Figure 9.10illustrates these sensitivities. It shows the typical high-, middle-, and low-cloud fractions over the tropical and subtropical oceans, as a function of the sea surface temperature and mid-tropospheric large-scale vertical velocity $\omega_{500}$ at $500 \mathrm{hPa}$ (in pressure coordinates so that the negative values correspond to ascent and the positive values to subsidence). It also shows the joint Probability Density Function (PDF) of SST and $\omega_{500}$. The high-cloud fraction exhibits little dependence on the SST, and it is almost linearly related to $\omega_{500}$, with large high-cloud fraction for strong ascent. Still, from the joint PDF it appears that strong monthly-mean ascent $\left(\omega_{500}<-30 \mathrm{hPa} /\right.$ day $)$ only occurs over warm SSTs, pointing to a role of SST gradients in maintaining deep convection and ascent. Precipitation exhibits the same sensitivity as the high-cloud fraction (not shown). Mid-tropospheric clouds exhibit roughly the same sensitivity as high clouds, except over cool SST, where midlevel clouds associated with extratropical intrusions can be observed.

Low clouds have the opposite sensitivity; low-cloud fraction decreases with increasing ascent: shallow convection is replaced by deep convection and high clouds mask the lowlevel clouds below them. Low-cloud fraction also increases with increasing subsidence, which shows that the effect of subsidence on LTS dominates that of ventilation. Unlike in ascending regions, low-cloud fraction is very sensitive to SST in subsiding regions.

The relationship between large-scale vertical motions and cloudiness shown in Figure 9.10 for monthly means is valid at shorter timescales in reanalyses and campaign measurements.

Although vertical advection is the predominant contribution, horizontal advection can be significant. For example, (i) cooling by horizontal advection in the eastern subtropical basins facilitates saturation and contributes to maintaining stratocumulus decks. (ii) Vertical shear can damp convection by transporting free-tropospheric moisture anomalies away from their convective source and canceling the moistureconvection feedback. (iii) Horizontal moisture transport is also thought to contribute to the propagation of the MJO.

\subsubsection{How clouds influence circulation}

The large-scale circulation of the atmosphere is well described by the quasi-static equations that result from applying the hydrostatic and anelastic approximations to the Navier-Stokes equations. Here, we will use these equations in pressure coordinates, as described in Chapter 2: the hydrostatic equation (2.93), and the mass, momentum, and energy budgets (2.98) to (2.101). Here we write the horizontal momentum equation (2.99) modified to include the Coriolis acceleration, and the continuity equation (2.98):

$\begin{aligned} \mathrm{D}_{t} \boldsymbol{v}_{p}+\mathbf{f} \times \boldsymbol{v}_{p} & =-\nabla_{p} \phi, \\ \nabla_{p} \cdot \boldsymbol{v}_{p}+\partial_{p} \omega & =0,\end{aligned}$

with $\boldsymbol{v}_{p}$ the horizontal wind in pressure coordinates, $\omega$ the pressure velocity, $\phi$ the geopotential, and $\mathbf{f}$ the Coriolis parameter vector. Equation (9.1) reveals that the geopotential gradient is the primary forcing of the horizontal flow. The other terms of the momentum flux (transport and Coriolis acceleration) are functions of the flow. And Equation 9.2 shows that, to ensure mass conservation, the vertical motion is the vertically integrated convergence of the horizontal flow.

So the key to the action of clouds on circulation is the modulation of the geopotential gradient by cloud processes. 

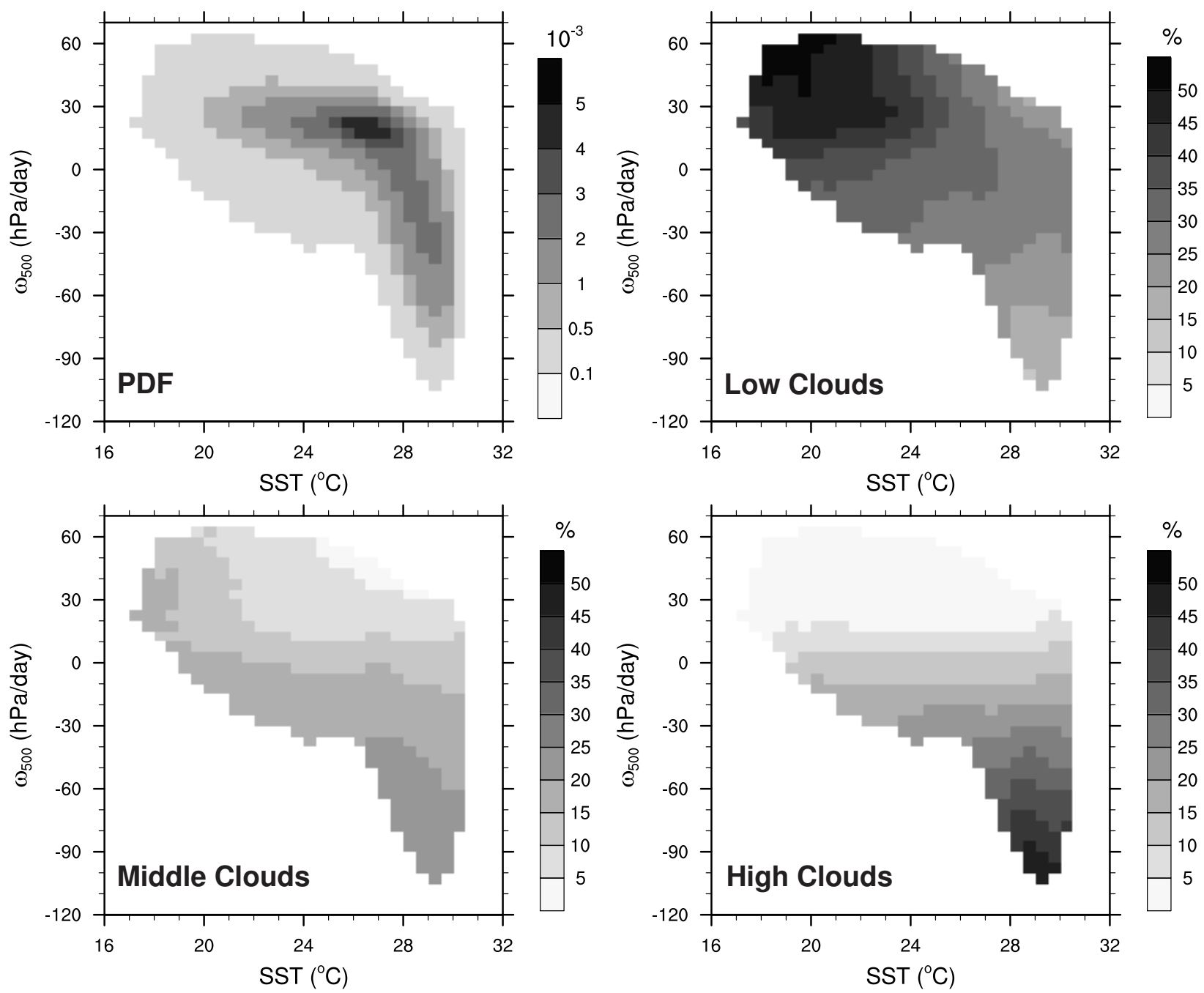

This modulation results from diabatic processes. In the tropics and subtropics, the hydrostatic approximation (2.93) and equation of state (2.6) can be combined as follows:

$$
\partial_{p} \phi=-\frac{R_{\mathrm{d}} T_{\rho}}{p}
$$

where $T_{\rho}=T\left(1+\epsilon q_{\mathrm{v}}-q_{\mathrm{c}}\right)$ is the density (or virtual) temperature with $q_{\mathrm{v}}$ the specific humidity, $q_{\mathrm{c}}$ the specific condensed water, and $\epsilon=R_{\mathrm{V}} / R_{\mathrm{d}}-1$, with $R_{\mathrm{v}}$ the gas constant for water vapor and $R_{\mathrm{d}}$ the gas constant for dry air.

Let's consider a cloud with its base at $p_{b}$ and its top at $p_{t}$. Equation 9.3 can be differentiated on an isobaric surface and integrated on the vertical to obtain an expression of the difference in horizontal geopotential gradient between the top and bottom of the cloud:

$$
\nabla_{p} \phi_{t}-\nabla_{p} \phi_{b}=R_{\mathrm{d}} \int_{p_{t}}^{p_{b}} \nabla_{p} T_{\rho} \frac{d p}{p},
$$

which shows that the change of the horizontal geopotential gradient across the depth of the cloud is proportional to a vertical integral of the density temperature gradient (it is the same equation that leads to the thermal wind shear relationship in the extratropics). Note that the contribution of the density temperature gradient at a given pressure level is

Figure 9.10 Probability Density Function of monthly mean $\omega_{500}$ and SST (ERA-Interim), and monthly mean cloudiness (ISCCP) as a function of $\omega_{500}$ and SST, over tropical and subtropical oceans $\left(30^{\circ} \mathrm{S}-30^{\circ} \mathrm{N}\right)$.

inversely proportional to the pressure. Now let's consider an atmosphere with uniform density temperature stratification except for this cloud which is warmer than the rest of the atmosphere. This corresponds to anomalies of geopotential smaller at the bottom of the cloud than at its top. Let's first assume that the geopotential anomaly is zero at the top of the cloud; in this case, we have a depression at the bottom of the cloud. In the absence of rotation, the geopotential gradients would essentially drive a converging horizontal flow below the cloud. With the Coriolis acceleration, the circulation is cyclonic and the convergent circulation is weaker (and with a spatially varying Coriolis parameter, the circulation response is not symmetric with respect to the geopotential perturbation ${ }^{6}$, but convergence occurs nonetheless. By continuity, convergence below the clouds corresponds to ascending motion within the cloud and, since this type of circulation does not penetrate significantly in the stratosphere, this implies divergence above the cloud. This is in contradic-

6 See Exercise 3 on the role of rotation and $\beta$ effect. 
tion to our assumption that the geopotential anomaly is zero above the clouds. Continuity in fact dictates that a weighted average of the geopotential anomalies at the top and at the bottom of the clouds is zero, so that the two anomalies have opposite signs. A warm cloud is associated with a geopotential minimum below and a geopotential maximum above, and therefore with convergence below the cloud and divergence above.

Rather than controlling temperature per se as in the simple example considered above, clouds contribute to diabatic heating via phase changes, radiative processes, and in-cloud mixing. The partial time derivative of Equation (9.4) yields the same integral linking the change in geopotential gradient to the tendency of density temperature gradient, and we can isolate the contribution of clouds:

$$
\partial_{t}^{\mathrm{cl}} \nabla_{p} \phi_{t}-\partial_{t}^{\mathrm{cl}} \nabla_{p} \phi_{b}=R_{\mathrm{d}} \int_{p_{t}}^{p_{b}} \nabla_{p}\left(\partial_{t}^{\mathrm{cl}} T_{\rho}\right) \frac{d p}{p},
$$

where $\partial_{t}^{\mathrm{cl}}$ indicates the contribution of the clouds to the local derivative. Strictly speaking, $\partial_{t}^{\text {cl }} T_{\rho}$ depends on the cloud heating and moistening, but the main contribution is the heating $\left(\partial_{t}^{\mathrm{cl}} T\right)$ and in the following discussion, we will focus on this term. Equation 9.5 describes how cloud diabatic heating can change the horizontal gradient of geopotential across the depth of a cloud. Our reasoning with a simple warm cloud above still holds, although with some delay effects. If a cloud warms the troposphere, the geopotential difference across the cloud increases and outward horizontal geopotential gradients are created below the cloud while inward geopotential gradients are created above the clouds. This forces convergence below the clouds and divergence above This clearly opens a positive convectioncirculation feedback: warming by a deep cloud results in ascent which is favorable to deep clouds, as shown in the previous section. As noted above, Coriolis acceleration limits convergence, so that this type of feedback is expected to be stronger in the deep tropics than in the subtropics. The latitudinal variation of the Coriolis parameter also breaks the symmetry of the circulation response with respect to the cloud diabatic pattern, and this could intervene in the propagation of cloud systems and their envelopes. Because clouds can vary on a wide range of spatial and temporal scales, the geopotential gradients created by the cloud diabatic effect can feed back on a large range of transient and steady features of the tropical climate.

As pointed out above, the contribution of the heating gradient to the geopotential gradient is inversely proportional to the pressure. Therefore, in order to represent properly the effect of clouds on the dynamics, it is not enough to simulate properly the vertically integrated heating; it is also necessary to simulate correctly the details of the profile of cloud heating. Biases of the profiles of diabatic heating are thought to be an important source of biases in general circulation model. At the same time, it is very difficult to extract reliable diabatic heating profiles from available observations and to discriminate between the different contributions to this heating. The lack of an observational basis limits our ability to constrain this aspect of model parameterizations. The vertically integrated heating is better constrained since the column latent heating is essentially proportional to the surface precipitation and the column radiative heating is given by the surface and top-of-the-atmosphere budgets.

\subsubsection{Cloud diabatic effect}

Clouds have a strong diabatic effect on the atmosphere through three main processes: phase change, radiation, and turbulent mixing due to cloud-scale circulations. Cloud formation releases latent heat by condensation and freezing. Melting and re-evaporation of precipitation cause latent cooling. Hydrometerors also interact with solar and terrestrial radiation: they scatter and absorb incoming radiation, and emit in the terrestrial range. A lot of mixing occurs inside convective clouds, due to updrafts in the cloudy convective plumes and downdrafts in precipitating convective clouds as well as in the vicinity of convective clouds.

The total diabatic heating can be estimated from campaign observations (GATE, TOGA-COARE, etc...) as a residual term from the dry static energy budget because storage and transport can be computed from the temperature and wind observations. Simulations of the cases corresponding to these campaigns can provide an estimate of the latent, radiative, and mixing contributions to this total heating. Some modern satellites such as TRMM provide radar observations of rainfall which can be used to estimate the latent heating, often using input from cloud-resolving models. The various existing methods yield estimates of vertical profiles that differ significantly. Estimates of radiative heating can be obtained combining satellite measurements and radiative numerical codes: the satellites provide cloud properties (e.g., ISCCP products) and the radiative budget of the Earth (provided by the Earth Radiation Budget Experiment - ERBE - and more recently by Clouds and Earth's Radiant Energy System -CERES - instruments) are used to constrain the numerical simulations. Extracting the cloud contribution to the radiative heating can be done by numerical experiments or by subtracting composites of clearsky observations from the all-sky observations. There is still some uncertainty on the vertically integrated estimates, and a lot of uncertainty on the details of profiles, and better documenting and understanding the vertical profile of mixing, latent, and radiative heating will continue to be a challenge in the years to come. Nevertheless, we presently have a rough idea of what type of heating profile is due to each cloud type.

Figure 9.11 shows these typical tropospheric profiles of diabatic heating due to these three processes (phase change, radiation, and mixing) for different cloud types (first row).

Typical surface precipitation rates are also indicated, and they are proportional to the vertically integrated latent heating. This figure is based on a compilation of data from observation campaigns, satellite retrievals (some using cloudresolving models), and weather-station soundings; it should be considered as illustrative rather than exact. If the vertical profiles of heating for convective clouds are fairly robust, the altitude of sign change and amplitude of the latent heating for stratiform precipitating clouds are less robust, and the radiative profiles are sensitive to a variety of factors, including aerosols. Figure 9.11 also shows the profiles 


\section{Stratocumulus}
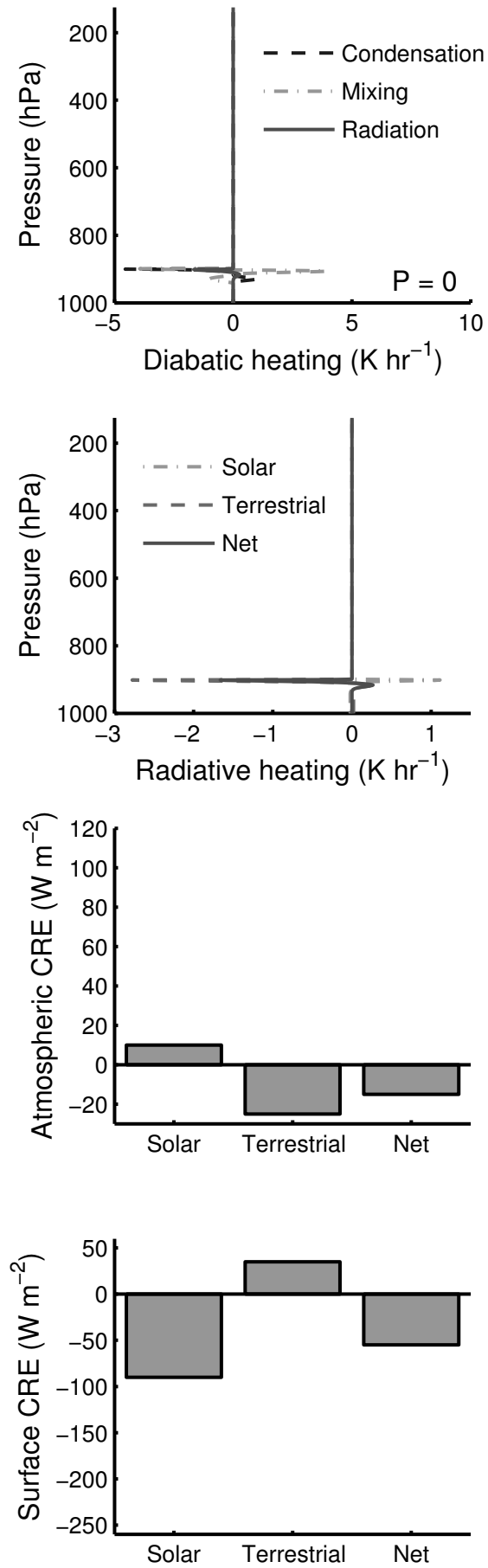

\section{Cumulus}
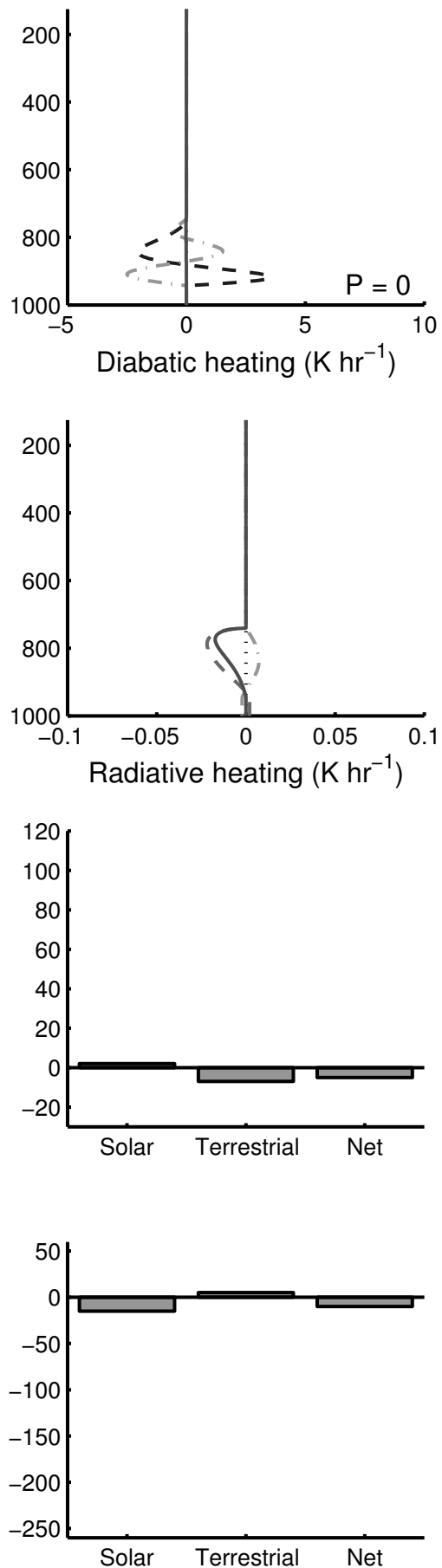

Congestus
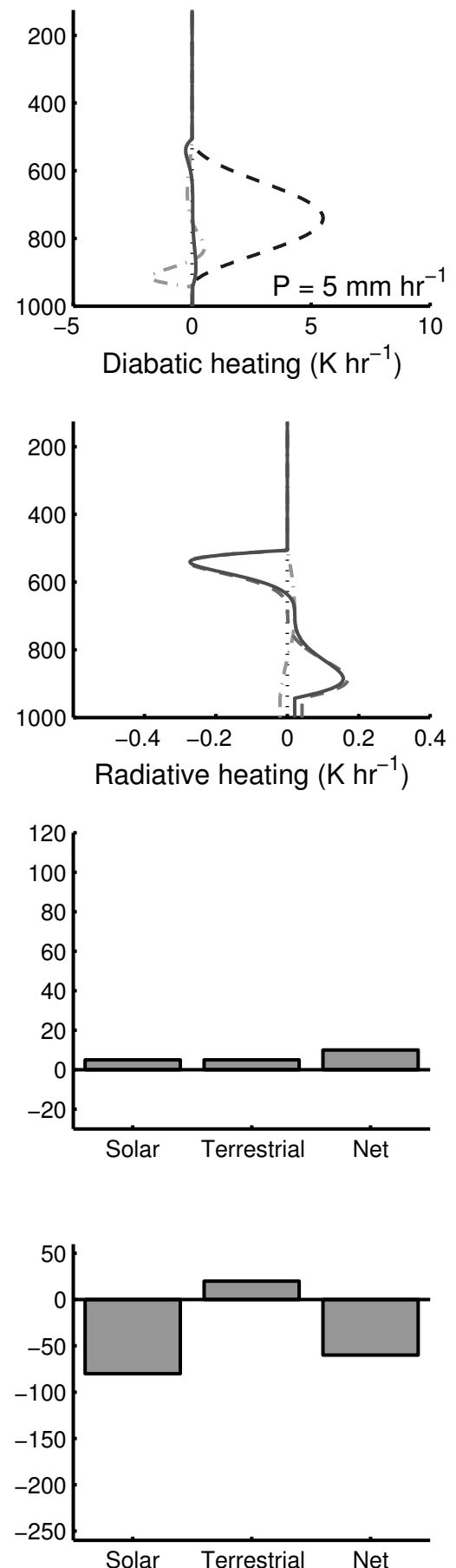

of solar and terrestrial contributions to the radiative heating (second row) and their vertical integral that corresponds to the radiative flux divergence across the atmospheric column (third row). Radiative effects are given for an average daily solar insolation, so depending on the time of the day of the cloud occurrence its radiative effect in the solar range can be significantly different. The main cloud type that is not represented in Figure 9.11 is the cirrus clouds which have the same heating properties as anvil clouds except over a thinner layer in the upper troposphere. We also chose to
Figure 9.11 Typical profiles of latent, turbulent, and net radiative heating (first row), and the solar, terrestrial and net radiative heating (second row), as well as total atmospheric (third row) and surface (fourth row) radiative heating derived from observation campaigns and satellite retrievals, associated with different types of clouds: stratocumulus, shallow cumulus, congestus, deep convective, stratiform precipitating, and anvil clouds. Typical precipitation rates are indicated in each panel on the first row. CRE stands for Cloud Radiative Effect. 

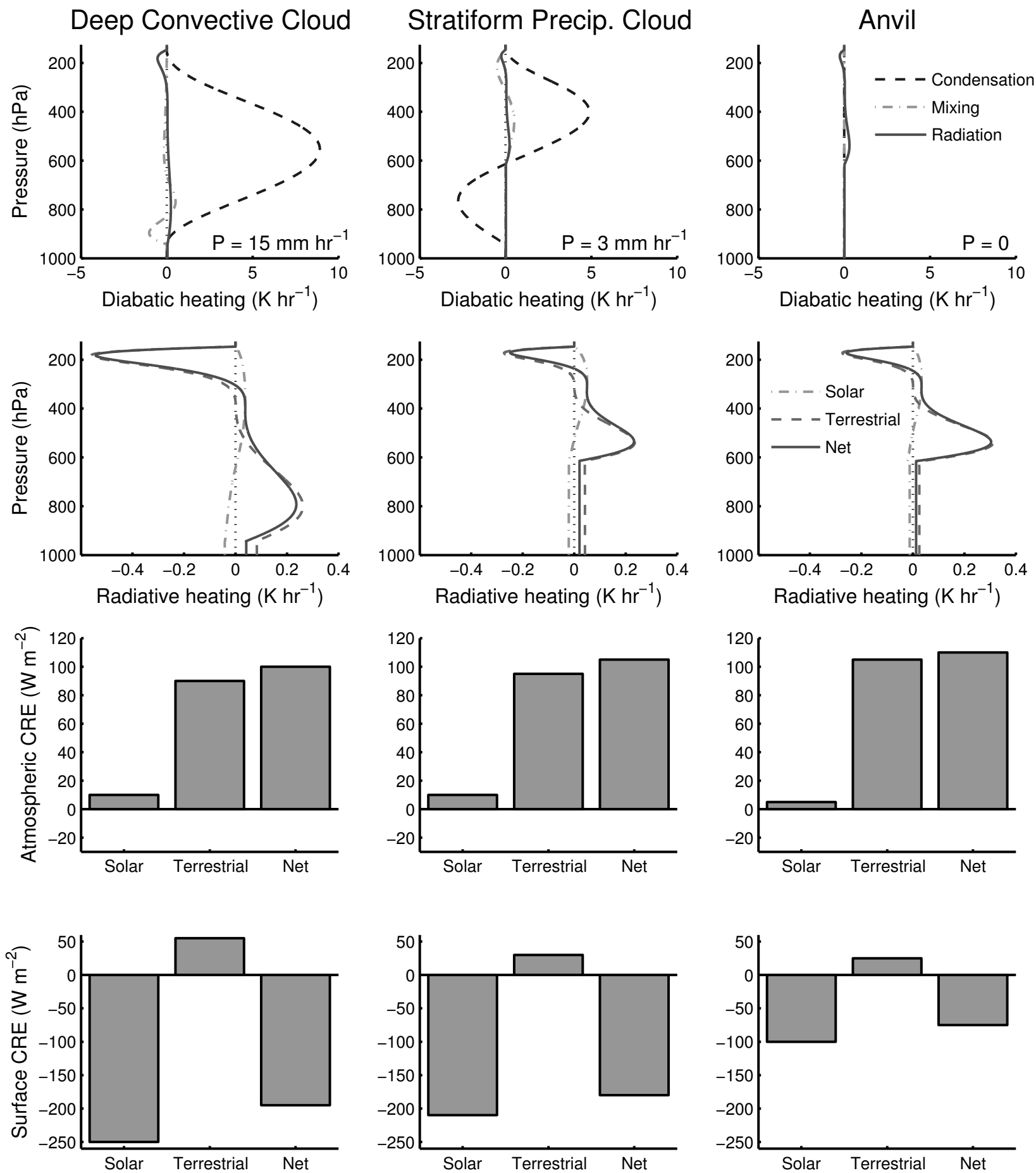

represent the profiles typical of non-precipitating stratocumuli and shallow cumuli (typical of the Barbados Oceanographic and Meteorological Experiment - BOMEX, 1969) even though stratocumuli can drizzle and a small percentage of cumuli, the deepest ones, do precipitate, as observed during the Rain In Cumulus over Ocean (RICO) campaign in the subtropical western Atlantic in 2004. The typical latent heating profile of these cumuli is in between that of a non-precipitating cumulus and that of a congestus. From the first row in Figure 9.11 it appears that the cloud latent effect is dominant for all precipitating clouds $(\mathrm{Cg}, \mathrm{DCC}$, and
Figure 9.11 (continued).

SPC) by one order of magnitude. Cloud mixing and latent heating have the same order of magnitudes in the boundary layer clouds, and cloud radiative effect has the same order of magnitude as the two other effects in stratocumuli.

In non-precipitating stratocumuli and cumuli, condensation occurs in the lower part of the cloud and evaporation occurs in the upper part. Precipitating convective clouds $(\mathrm{Cg}$ and DCC) release latent heat with a maximum in the middle of the cloud. Stratiform precipitating clouds release latent 
heat in the cloud, and part of the precipitation is reevaporated between the cloud base and the surface, so that there is latent cooling from the surface to the cloud base. The non-precipitating anvil clouds are fairly passive, with little condensation.

Mixing is typically negative in the lower part and very top of the cloud, and positive in the upper part. The heat released by condensation in the lower part of the clouds is transported upwards by cloud turbulence and warms the upper part of the cloud (that is cooled by evaporation). At the very top of the cloud, the turbulent overshoots tend to cool the layer, particularly when a stable layer, such as an inversion, caps the cloud. The respective magnitudes of these patterns depend on the cloud type. The cooling by mixing at the base of the cloud is clear for all types of boundarylayer and convective clouds ( $\mathrm{Sc}, \mathrm{Cu}, \mathrm{Cg}$, and $\mathrm{DCC}$ ), but the other features are significant only in boundary-layer clouds (Sc and $\mathrm{Cu}$ ).

The atmospheric cloud radiative effect (CRE) is small compared to the other terms except in the case of the stratocumuli, at the top of which radiative cooling is a major term in the energy budget. The second and third rows of Figure 9.11 further detail the solar CRE and the terrestrial CRE. The shortwave CRE is due to the absorption of incoming solar radiation by clouds (possibly after multiple scattering), and its vertically averaged atmospheric contribution is generally positive (i.e., a warming), roughly increasing with the optical depth of the cloud. Most of the absorption occurs in the upper part of the cloud. Below this absorption layer, the SW CRE can be negative because the downward SW radiative flux is reduced by the absorption and scattering above, so that the local absorption, that is proportional to this incoming flux, is reduced. The longwave CRE results from a competition between increased emission (that is a function of temperature) and increased absorption (that is proportional to the incoming fluxes) due to the presence of cloud hydrometeors. It is positive in the lower part of the cloud where absorption dominates, and negative in the upper part of the cloud where emission dominates. The net vertically integrated contribution depends strongly on the cloud type, mostly because of the temperature at cloud top: moderate cooling for stratocumuli, small cooling for cumuli, small warming for congestus clouds, and a large warming for clouds with high tops (DCC, SPC, An).

The variety of heating profiles associated with different cloud types clearly shows the challenge that parameterization developers face. Parameterizations need to simulate the effect of a population of clouds with distinct characteristics in order to obtain the correct cloud-driven circulation. As an example, we can compare the circulation caused by the latent heating from a deep convective cloud versus that caused by a stratiform precipitating cloud. The DCC warms the troposphere with a maximum in the mid-troposphere, creating density temperature gradients between the cloud and the environment; this results in outward geopotential gradients with increasing amplitude downward. Horizontal convergence with a maximum at the surface and ascent in the cloud will ensue. The SPC warms the upper troposphere and cools the lower troposphere; because the vertically in- tegrated heating is positive (i.e., the surface precipitation is positive) and because the elevated heating is more efficient at creating geopotential gradients due to the weight $1 / p$ in the integral, the SPC diabatic heating will create horizontal convergence over the whole troposphere like the DCC, even at the surface. But unlike in the case of the DCC, the maximum convergence will occur in the mid-troposphere, where the SPC heating changes sign. Furthermore, for a given precipitation rate and cloud area, the overturning circulation resulting from SPCs will be much larger than the circulation resulting from DCC: 7 Simulating the correct proportion of convective and stratiform rain is currently a challenging issue in general circulation models.

It would certainly be interesting to show a figure similar to Figure 9.11 for cloud moistening profiles. Cloud moistening is crucial for its direct effect on moisture stratification rather than for its effect on circulation through density temperature gradients. Unfortunately, there is even more uncertainty on these moistening profiles than on heating profiles.

Figure 9.12 shows the climatology of cloud radiative effect vertically integrated over the atmosphere, as well as the column-integrated latent heating (estimated by multiplying the surface precipitation by a constant latent heat of vaporization $L_{\mathrm{v}}$ ). The climatologies of cloud radiative effect are related to the values in Figure 9.11 (last two rows) by the climatological frequency of occurrence of the different cloud types.
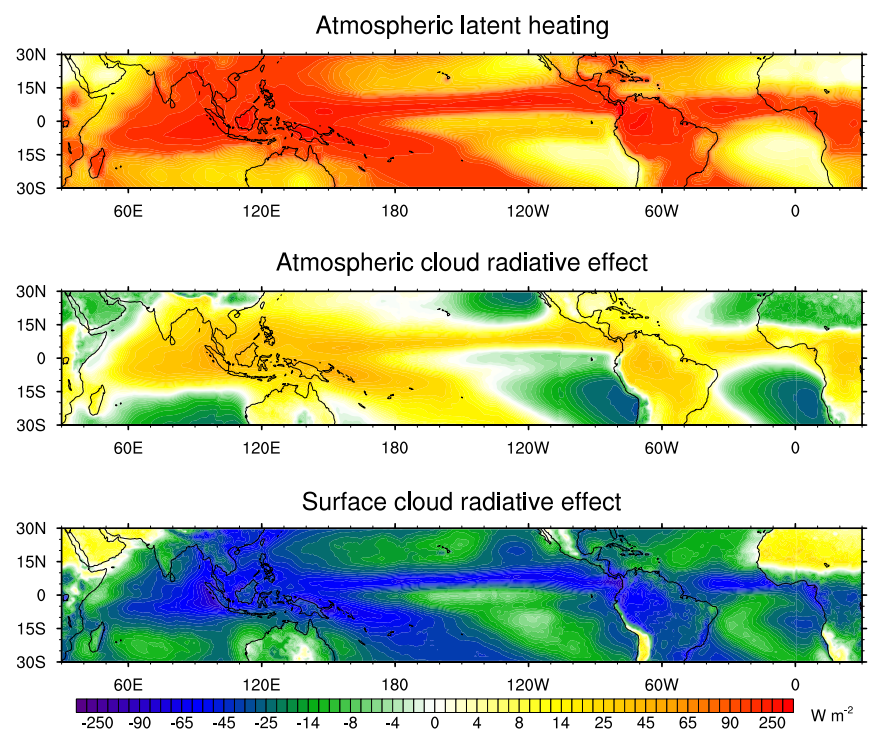

Figure 9.12 Vertically integrated latent heating of the atmosphere, atmospheric cloud radiative effect, and the surface cloud radiative effect. Data from NASA's Surface Radiation Budget (SRB) Project for the period 1998 to 2007.

In convergence zones (ITCZ, SPCZ, WPCZ), the columnintegrated diabatic heating is overwhelmingly dominated by latent heat release in precipitating clouds (typically 250 $\mathrm{W} / \mathrm{m}^{2}$ ). Initially, the precipitation was thought to occur mostly under deep convective clouds, but campaigns such as

7 Exercise 2 illustrates these points in the case of the Walker circulation. 
TOGA-COARE showed that the congestus clouds account for about a quarter of the precipitation in CZs, and satellite data (TRMM) have shown that stratiform clouds account for almost half the surface precipitation. This is surprising considering that the rain rates associated with each cloud type $(\mathrm{Cg}, \mathrm{DCC}$, and SPC typical rain rates: $5 \mathrm{~mm} / \mathrm{hr}, 15$ $\mathrm{mm} / \mathrm{hr}$ and $3 \mathrm{~mm} / \mathrm{hr}$, see Figure 9.11) suggest that the deep convective clouds precipitate much more. In fact, deep convective clouds are very infrequent compared to congestus and stratiform precipitating clouds. For example, within an MCS, the area covered by stratiform clouds is 5 to 10 times larger than that covered by deep convective clouds.

In convective regions, the net atmospheric cloud radiative heating is about five times smaller than the latent heating (about $50 \mathrm{~W} / \mathrm{m}^{2}$ ). The net CRE in these regions is dominated by the large terrestrial warming associated with deep clouds. Over the ocean, the solar CRE is a small warming, but over continents and islands, it can be a small cooling, because of the reduced absorption in the lower troposphere. In the trade-wind, shallow-convective regions, small cumuli exert a moderate radiative cooling on the atmospheric column, which has roughly the same magnitude as the latent heating resulting from occasional deep convective events or the deepest shallow cumuli, resulting in near-zero net diabatic heating. In coastal regions west of the subtropical continents, stratocumuli have a significant cooling effect (about $-30 \mathrm{~W} / \mathrm{m}^{2}$ ) resulting from strong terrestrial cooling (about $-40 \mathrm{~W} / \mathrm{m}^{2}$ ) at the top of the cloud that is hardly modulated by a small solar warming. The column-integrated latent heating is negligible in these regions (very little drizzle reaches the surface).

Overall, the climatological CRE enhances the diabatic heating contrasts between precipitating and dry regions; the $\mathrm{CRE}$ warms the atmosphere in the CZs and cools the atmosphere in convectively-suppressed regions populated by boundary-layer clouds ( $\mathrm{Sc}$ and $\mathrm{Cu}$ ).

\subsubsection{Idealized frameworks}

Equation 9.5 is a diagnostic tool and it cannot be used to study the convection-circulation feedback loop. On the other hand, the quasi-static equations are a closed mathematical system so complex that it is difficult to use them to grasp the fundamental interplay between moist physics and dynamics. Consequently, a number of approaches have been developed to simplify the problem and study interactions and feedbacks in idealized frameworks. The most basic of these approaches focus on the interaction between vertical motion and moist physics in an atmospheric column and essentially parameterize the large-scale vertical velocity.

The first of such approaches is called the Weak Temperature Gradient (WTG) approximation. It relies on the observation that gravity waves rapidly damp the free-tropospheric horizontal gradients of temperature, and as a result the horizontal transport of energy is negligible. This approximation is used to simplify the thermodynamic equation (2.100) in the free troposphere, which can be written as follows in steady state:

$$
\omega \partial_{p}\left(c_{e} T+\phi\right)=\nabla \cdot \boldsymbol{q}+Q_{\mathrm{c}}
$$

The right-hand side of this equation is the diabatic heating, with $\boldsymbol{q}$ the total heat flux (due to radiation and mixing) and $Q_{\mathrm{c}}$ the net condensation heating. If the free-tropospheric profile of temperature is known, Equation 9.6 provides a parameterization of the pressure velocity $\omega$ that sets it proportional to the diabatic heating: a warming causes collocated ascent, and a cooling causes subsidence. The pressure velocity is then used to compute the vertical transport of the other variables. The horizontal transport of variables other than temperature has to be imposed or parameterized. A relaxed version of this approximation has also been proposed: the large-scale vertical motion is assumed to relax the free-tropospheric temperature profile towards a reference profile. This formulation is particularly useful in cloud-resolving models, in which Equation (9.6) cannot be implemented. These WTG and relaxed WTG approximations have proven useful to understand the very basic interaction between moisture transport and cloud physics. Still, the collocation of diabatic heating and vertical motion stipulated in this approach might be too local in the light of Equation 9.5 that establishes the dependence of geopotential on heating. Horizontal convergence thus depends on the vertical profile of heating and vertical motion, through the continuity equation, has a non-local relationship to the heating.

Another idealized approach does take into account this non-local relationship. It is called the Damped Gravity Wave (DGW) approximation because it uses a steady wave solution to the damped, quasi-static momentum equation with a motionless basic state and no rotation. This simplified momentum equation can be written:

$$
0=-\nabla_{p} \phi-\eta \boldsymbol{v}_{p}
$$

which is essentially a linearized, steady Equation (9.1) without Coriolis acceleration and with an additional damping term; $\eta$ is the damping rate. Wave solutions with wavevector $\boldsymbol{k}:\left(\boldsymbol{v}_{p}, \omega, \phi, T_{\rho}\right)=\left(\hat{\boldsymbol{v}}_{\boldsymbol{p}}, \hat{\omega}, \hat{\phi}, \hat{T}_{\rho}\right) e^{i \boldsymbol{k} \cdot \boldsymbol{x}}$, with $\boldsymbol{x}$ the horizontal space vector, to Equation (9.7) together with the continuity and hydrostatic equations 9.2 and 9.3 , verify ${ }^{8}$

$$
\partial_{p}\left(\eta \partial_{p} \hat{\omega}\right)=\frac{R_{\mathrm{d}}}{p} \boldsymbol{k}^{2} \hat{T}_{\rho}
$$

Using this equation, we can compute the pressure velocity $\omega$ that results from a perturbation of $T_{\rho}$ from a reference profile, using $\omega=0$ as boundary condition at the surface and at the tropopause. This pressure velocity is then used to compute the vertical transport of thermodynamic variables (including $T$ ), and the horizontal transport has to be imposed or parameterized. Compared to the WTG approximation, the DGW approximation reintroduces a complex relationship between the perturbation of the temperature profile (resulting from diabatic heating) and the profile of vertical motion, as shown by the double derivative in Equation 9.8). The main simplification in this approach is that the horizontal momentum is exactly proportional to

8 Details of the derivation are the object of Exercise 1. 
the geopotential gradients. This implies that the horizontal convergence is collocated with the Laplacian of the geopotential. In wave solutions, the Laplacian is proportional to the variable, so that the horizontal convergence is collocated with the geopotential perturbation in the DGW approximation. A more complex approximation named Weak Pressure Gradient (WPG) approximation follows a similar approach, but it uses the general solution of linearized, time-dependent Boussinesq equations.

The potential and limits of these idealized approaches are still being investigated. They already provide an opportunity to study the interaction between moist physics and dynamics in an atmospheric column, providing a more tractable framework than the full tri-dimensional equations over a large horizontal domain. This type of approach can be used in realistic or idealized case studies, implemented in cloud-resolving or single-column models. They have not been widely used so far for model improvement, but the comparison between single-column models and cloud-resolving models using this type of representation of the large-scale circulation is seen as a promising avenue.

\subsubsection{Cloud-circulation interaction and climate}

The convection-circulation feedback is active in all the major features of the tropical climate and their variability. It is one of the crucial mechanisms that produce cloud systems and their envelopes: as deep convection concentrates in a cloud clusters, a circulation develops in between the heated convective regions and the radiatively-cooled dry regions. This circulation transports moisture from the dryregion boundary layer to the convective clusters, fuelling convection in these regions, and drying the non-convective free troposphere via downward transport in the dry regions, inhibiting deep convection there.

The cloud diabatic heating in the ITCZ intensifies the Hadley circulation, which would exist in the absence of cloud processes just from the differential solar heating between the equator and the subtropics. This intensification is particularly strong when the ITCZ is away from the equator according to the angular-momentum theory of the Hadley circulation; this is one of the mechanisms that maintains the ITCZ north of the equator rather than on the equator where the solar forcing is maximum (see Figure 9.13). Some model experiments even suggest that the interaction between clouds and circulation is enough to break the interhemispheric symmetry and locate the ITCZ away from the equator. The cloud diabatic effect in monsoon CZs is even further from the equator than in the case of the ITCZ, and this contributes to the strength of monsoon circulations. Because turbulent heat fluxes increase with the surface wind, they feed back on the circulation: a more intense circulation means larger surface winds and larger surface latent and sensible heat flux; this additional energy is transported by the circulation to the ITCZ region and intensifies deep convection there.

The convection-circulation feedback also reinforces the Walker circulation, which is primarily forced by orography and land-sea contrasts, and its main form of interannual variability (ENSO), which is a coupled ocean-atmosphere mode. The cloud diabatic heating in the ascending branch of this circulation creates zonal gradients of geopotential that sustain convergence in these ascending branches. When the Walker circulation is perturbed during an El Niño event, the cloud diabatic effect causes ascent in the central equatorial Pacific and strengthens the eastward shift of the West Pacific CZ (see Figure 9.14)

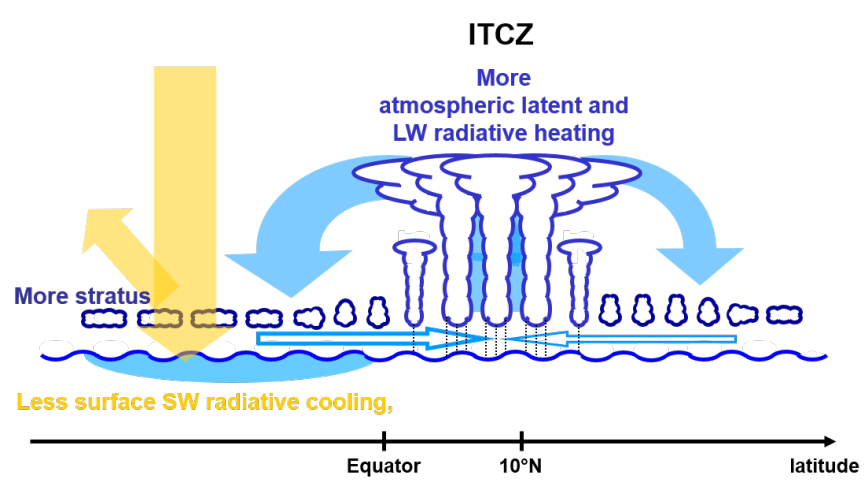

Figure 9.13 Schematic of the ITCZ and the associated cloud mechanisms: (i) the convection circulation feedback enhances the ITCZ in its off-equatorial location; (ii) SST-stratocumulus feedback cools the SST south of the equator; (iii) the near-surface circulation is enhanced by the cross-equatorial SST gradient; (iv) the surface winds also enhance surface turbulent heat fluxes and upwelling south of the equator, resulting in additional cooling of the ocean surface there.

The issue of the vertical profile of cloud diabatic heating, through the factor $1 / p$ in Equation (9.5), was raised as early as the 1980s in the context of the simulation of the Walker circulation: it was shown that a top-heavy vertical profile of diabatic heating (associated with the presence of clouds with a significant fraction of stratiform precipitation) induces a stronger Walker circulation in better agreement with the observations than a profile that exhibits a maximum heating in the mid-troposphere (as that induced by deep convective clouds ${ }^{9}$ Later, TRMM confirmed this deduction by showing that almost half the surface precipitation originates from stratiform precipitating clouds. More recently, observations in the East Pacific and Atlantic have revealed a shallow meridional overturning circulation with outflow in the lower free troposphere (associated with a bottom-heavy profile of diabatic heating) additional to the troposphere-deep canonical Hadley circulation. These observations point to a complex latitudinal distribution of diabatic-heating profiles in these regions.

The circulation associated with the MJO is similar to the quasi-steady dynamical response following the cloud diabatic forcing. In this circulation pattern, known as the Gill circulation, large-scale ascent is almost co-located with the diabatic heating, and the resulting atmospheric transport is a strong positive feedback on the MJO convective disturbance. This convection-circulation feedback is thought to be essential to the development of an MJO event. Also,

9 This is illustrated in Exercise 2. 


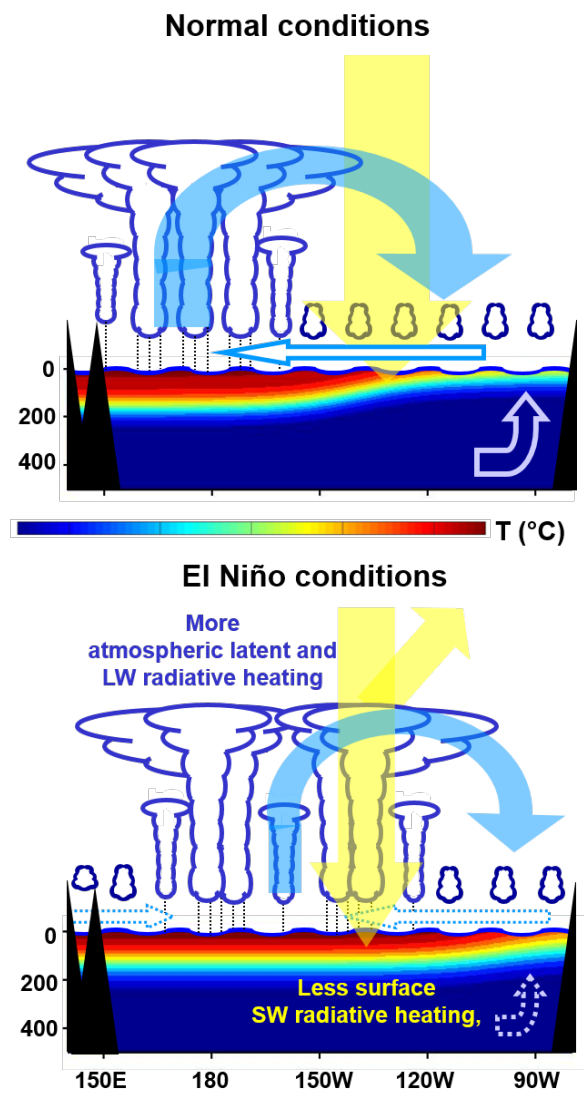

Figure 9.14 Schematic of the normal and El Niño conditions and the associated cloud mechanisms: (i) the positive Bjerknes feedback tends to maintain the El Niño (or normal, or La Niña) conditions through the interaction of the SST gradients, surface winds, and upwelling; (ii) the SST-high cloud and the SST-flux negative feedbacks are responsible for the return to normal conditions.

more ascent occurs east of the maximum of diabatic heating than west of it. This should moisten the lower free troposphere and favor the eastward propagation of deep convection (see Figure 9.15). As pointed out above, the leading hypotheses to explain the difficulties encountered in modeling the MJO are errors in the profile of diabatic heating, or the lack of large-scale organization of deep convective clouds. Both shortcomings likely yield a wrong dynamical response and prevent the development and propagation of an MJO event.

\subsection{Cloud-surface interaction}

Clouds are sensitive to surface conditions because these constrain the surface turbulent fluxes of energy and humidity, which affect the boundary-layer temperature and humidity and thus the stratification of the atmospheric column. Also, the surface turbulent buoyancy flux conditions the intensity of turbulence in the boundary layer and therefore its deepening or thinning and the boundary-layer cloud type. Clouds are thus sensitive to the sea surface temperature over the

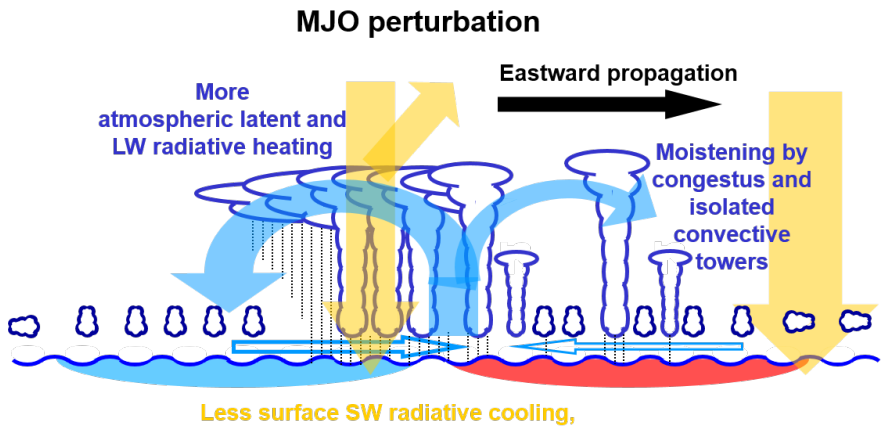

Figure 9.15 Schematic of an MJO perturbation and the associated cloud mechanisms: (i) the convection-circulation positive feedback is crucial to the development of the MJO event; (ii) the strong westerlies under the convective disturbance cause large turbulent surface heat fluxes that are also instrumental in maintaining the disturbance; (iii) together with this wind-flux feedback, the SST-high cloud negative feedback creates an SST gradient across the convective disturbance that enhances its eastward propagation through the SST-flux effect and the free-tropospheric moistening east of the disturbance.

oceans and they are sensitive to multiple factors over land: the vegetation type, land characteristics, soil temperature and moisture.

Reciprocally, by interacting with radiation, clouds change the downwelling flux at the surface and generally cool the surface. Clouds also affect the surface winds locally through convective gustiness and at larger scales by the change of circulation discussed in the previous section. Since turbulent surface fluxes increase with surface wind speed, these changes in wind can affect the surface conditions. Precipitating clouds also change the soil moisture content over the continents, which can also change vegetation type and associated characteristics (albedo, exchange coefficients) over long time scales. Here, we will focus on the oceanic surfaces; discussion on the land surfaces can be found in Chapter 12 .

\subsubsection{Influence of sea surface temperature on clouds}

Both the surface sensible heat flux and the latent heat flux increase with increasing SST. This warms and moistens the boundary layer and increases the conditional instability of the atmospheric column. It also increases the turbulence in the boundary layer and deepens it. As a result, we expect cloudiness to be sensitive to SST.

Figure 9.10 shows the sensitivity of cloudiness to the underlying SST. As noted in section 9.4.1 high clouds and precipitation do not exhibit a strong intrinsic sensitivity to SST, except that intense precipitation occurs mostly in regions of ascent over warm SSTs, and the occurrence of large-scale ascent exhibits a strong increase for SSTs exceeding $27^{\circ} \mathrm{C}$. However, this threshold should not be considered as an absolute threshold, as its value depends on the tropical-mean SST. Middle-cloud fraction exhibits roughly the same sensitivity, except some mid-level clouds associated with extratropical intrusions, over low SSTs in neutral and subsiding regions. 
Low clouds are more sensitive to SST than higher clouds, with large cloud fractions up to $50 \%$ associated with stratocumuli over low SSTs in subsiding regions, and small cloud fractions associated with cumuli over warmer SSTs. A large part of this sensitivity is directly related to the lowertropospheric stability: the cooler the SST, the larger the LTS, and the thinner and moister the boundary layer. In a very stable lower troposphere, shallow convection is inhibited and stratocumuli develop. As the SST increases, the lower troposphere becomes less stable and the surface buoyancy flux increases; both factors are conducive to shallow convection.

Sea surface temperatures can influence cloudiness indirectly through the effect of their gradient on circulation: SST gradients are communicated to the air temperature in the boundary layer by the sensible heat flux, and geopotential gradients result from air temperature gradients following Equation 9.4 and force atmospheric circulations from the regions of cool SST to regions of warm SST. These circulations influence cloudiness as described in section 9.4.1 At the seasonal scale and on longer timescales, direct and indirect effects of the SST on cloudiness have similar effects: ascent and warm SSTs are generally collocated and both favor deep clouds, while subsidence and cool SSTs are also collocated and favor boundary-layer clouds.

\subsubsection{Clouds and the surface energy budget}

If clouds are sensitive to the surface temperature, they also have a strong effect on the surface energy budget. Clouds are responsible for a large part of the global albedo because of backward scattering on cloud hydrometeors, and this is particularly felt at the surface. The last row of Figure 9.11 shows the cloud radiative effect on the surface, for a surface albedo typical of the ocean. Conversely to the atmospheric CRE (third row), the surface CRE is dominated by the solar contribution, and it is a cooling effect. The terrestrial CRE is a small warming due to the cloud additional greenhouse effect. The magnitude of both the solar and terrestrial contributions are essentially a function of their optical depth: they are small for the optically-thin cumuli, moderate for stratocumulus, congestus, and anvil clouds, and large for deep clouds (DCC, SPC). The magnitude of the net surface $\mathrm{CRE}$ is sufficient to influence the surface temperature significantly. This cooling effect opens the way for a negative feedback between convection and SST and a positive feedback between low clouds and SST (all clouds cool the SST, which is unfavorable for deep, high clouds and favorable for low-cloud cover).

The bottom panel of Figure 9.12 shows the climatology of cloud radiative effects at the surface. In the CZs, the net radiative effect is a cooling of a similar magnitude as the radiative warming of the atmospheric column above (about $50 \mathrm{~W} / \mathrm{m}^{2}$ ). This net cooling is due to the strong solar contribution (up to $100 \mathrm{~W} / \mathrm{m}^{2}$ ) associated with the additional albedo of high, deep clouds, partly compensated by the terrestrial contribution due to the additional cloud greenhouse effect.
In the oceanic, shallow-convective regions, the net radiative effect due to optically-thin cumuli is close to zero because the solar cooling and terrestrial warming compensate (both are about $30 \mathrm{~W} / \mathrm{m}^{2}$ ). Under the optically-thick stratocumuli, the net cloud effect at the surface is a moderate cooling (about $30 \mathrm{~W} / \mathrm{m}^{2}$ ) that results from strong solar cooling (up to $100 \mathrm{~W} / \mathrm{m}^{2}$ ), modulated by significant terrestrial warming.

Over subtropical deserts, in particular the Sahara, the net cloud radiative effect at the surface is a warming due to the terrestrial contribution. Because the albedo of the desertic surfaces is large, the surface absorbs a limited fraction of the incoming solar radiation and the impact of clouds on incoming radiation is not felt as strongly as over regions of smaller surface albedo.

Clouds also affect the surface heat budget indirectly through their influence on surface turbulent fluxes. By ventilating and drying the subcloud layer, shallow and deep convection increase the evaporation and cool the surface. Convective downdrafts also cool the subcloud layer and this cooling is transmitted to the surface through an increase in sensible heat flux. Radiative cooling of the stratocumuluscapped boundary layer also increases turbulence and turbulent surface fluxes. Finally, clouds also impact the turbulent surface heat fluxes; because these fluxes increase with surface wind and sea-air temperature difference, local effects such as cold pools and convective gustiness enhance turbulent surface heat fluxes; changes in large-scale surface wind resulting from remote cloud diabatic effects can also modulate the SST via changes in these fluxes.

Clouds have multiple other indirect effects on the surface temperature through changes in oceanic mixing and circulation: precipitation on the other hand can locally restratify the surface and reduce mixing by injecting fresh water with low density at the surface. On the other hand, turbulent surface heat and momentum fluxes power the turbulence in the oceanic mixed layer, and therefore the entrainment of subsurface waters into this layer, so changes in surface wind and air-sea difference due to the clouds impact the oceanic mixing. The momentum flux also forces the large-scale oceanic circulation, which results in oceanic energy transport that influences the SST.

\subsubsection{Cloud-ocean interaction and climate}

Interactions between clouds and the oceanic surface are at play in many features of the tropical climate and its variability. The off-equatorial location of the ITCZ is partly explained by its proximity to the latitudinal maximum of SST north of the equator: warm SST permits the development of high clouds and precipitation, while cool SSTs south of the equator in the Eastern Pacific and Atlantic oceans inhibit deep convection and maintain stratocumulus decks off the coast of Peru and Namibia. The off-equatorial maximum of SST and precipitation results from the interaction between clouds, circulation and SST. The SSTstratocumulus feedback illustrated in Figure 9.13 is the direct positive feedback between SST and low clouds mentioned above: the large cloud fraction associated with stra- 
tocumulus decks in subtropical eastern basins yields a strong cooling effect on the surface ocean. As shown in section 9.5.1 cloud fraction increases with decreasing SST and the resulting positive feedback between stratocumuli and SST prevents the development of any deep convection south of the equator. Another indirect feedback involving circulation has been documented. Cloud diabatic heating in the ITCZ and the south-north SST gradient create a southward low-level geopotential gradient which forces a monsoon-like anomalous circulation: enhancement of the easterly trade winds south of the equator, northward cross-equatorial wind, and weakening of the trade winds north of the equator. Because the surface turbulent heat fluxes depend on the surface wind, they are reduced north of the equator and increased south of the equator, which reinforces the north-south SST contrast: this is called the wind-flux-SST feedback. These feedbacks might be enough to break the inter-hemispheric symmetry and locate the ITCZ in the northern tropics by themselves; in the real world, the geography of the western coasts of the Americas and Africa play a role via coastal upwelling, and current orbital parameters (set by the phase of the precession of the equinoxes) might also have an influence.

The negative feedback between high clouds and SST is active in the El Niño - Southern Oscillation, as well as an indirect feedback between SST, atmospheric circulation and oceanic circulation (see fig. 9.14). After a westerly wind burst in the equatorial West Pacific triggers an El Niño, the reduced east-west SST gradient and anomalous cloud atmospheric heating gradient weaken the equatorial easterly winds, which reduces the surface easterly current and the upwelling in the eastern Pacific. This in turn reduces the east-west SST gradient and the gradient of the thermocline depth and constitutes a positive feedback on the El Niño conditions called the Bjerknes feedback (note that this feedback is also active in maintaining the normal conditions). The return to normal conditions is due to oceanic equatorial waves and to surface fluxes in the Central and East Pacific, with two contributions: an increase in surface turbulent heat fluxes resulting from the increased SST constitutes a negative SST-flux feedback, and a decrease of incoming solar radiation at the surface due to the high-cloud radiative effect at the surface constitutes the negative SSThigh cloud feedback. The contribution of the latter is dominant in the return to normal conditions.

The negative SST-high cloud feedback is also active in the MJO: the convective disturbance cools the surface as well, but with a different outcome because of the propagative nature of this mode. As shown in Figure 9.15 the SST cools below the convective perturbation, so that along the propagation the SST is warm east of the convective perturbation and cool west of it. This tends to increase surface sensible and latent heat fluxes east of the convective perturbation (and decrease fluxes to the west) and favor the eastward propagation of convection, by an SST-flux mechanism. An indirect effect of the clouds on the surface, through circulation, is thought to be important for the MJO as well: the Gill circulation associated with the MJO is characterized by a strong westerly jet just west of the maximum of deep convection. This jet increases surface turbulent heat fluxes that fuel the MJO convective disturbance by a wind-flux mechanism, which also cools the surface and enhances the SST contrast across the convective disturbance.

\subsection{Concluding remarks}

Through their interaction with atmospheric circulations and the surface, clouds are essential contributors to the climatology and the climate variability in the tropics. While clouds have long been seen as passive expressions of tropical variability, we now increasingly recognize their active role in controlling this variability. Consistently, while clouds have long been characterized through their morphology or physical properties, we now realize that they should also be considered as "energetic bodies" in the climate system.

Understanding the physical mechanisms through which tropical clouds interact with the large-scale climate remains a challenge. One of the key mechanisms is the interaction between large-scale circulation and convective clouds. Better understanding the dependence of cloudiness on large-scale conditions, in particular on free-tropospheric humidity, is necessary to parameterize convection and understand the effect of large-scale transport on the cloudiness. Reciprocally, better documenting the profile of cloud diabatic effect is crucial to understand the influence of clouds on the circulation. Another key mechanism is the interactions between clouds and the surface at the large scale. In particular, studying interaction between clouds, circulation, and a heterogeneous surface is a challenging topic in need of creative approaches and frameworks.

This understanding is all the more necessary since clouds are still poorly represented in many state-of-the-art models. Climate models often exhibit some systematic flaws in the simulation of clouds, such as the double ITCZ syndrome, a weak MJO, or an underestimation of stratocumulus cover in the eastern subtropical basins. In terms of climate projections, the models do not agree on the sign of the predicted precipitation change during the $21^{\text {st }}$ century over most of the tropical continents, and tropical low clouds are a major contributor to the spread in predicted global-mean temperature change. A better understanding of cloud-climate interactions in the tropics will help improve the parameterizations of moist physics and to remediate these biases and uncertainties.

Fortunately, new opportunities arise to advance this understanding. New observations are becoming available (e.g., cloud latent heating and cloud radiative effect estimates from satellite observations). Large-domain high-resolution modeling (with cloud-resolving models and large-eddy simulations) is now computationally affordable and opens perspectives in the study of the interaction of clouds and the large-scale environment (e.g., the large-scale organization of convection). Finally, the scientific communities working on fine-scale cloud processes and large-scale climate studies are increasingly collaborating, opening new scientific perspectives. 


\section{Further reading}

The review article by Houze on "Mesoscale convective systems" (Review of Geophysics, 2004) is a good reference. On convectively-coupled equatorial waves, another review article is very detailed ("Convectively coupled equatorial waves", Kiladis et al., Review of Geophysics, 2009). Most aspects of the tropical intraseasonal variability are explained in the book "Intraseasonal variability in the ocean-atmosphere climate system" (Eds. Lau and Waliser, 2005); see also the review article by Zhang on the "Madden-Julian Oscillation" (Review of Geophysics, 2005). General elements on tropical waves and dynamics can be found in Chapter 11 of "Atmosphere-Ocean Dynamics" (Gill, 1982). Mapes et al. (Dynamics of atmospheres and oceans, 2006) introduces the concept of the stretched building blocks for large-scale modes of tropical variability. An extensive overview of the Hadley circulation is given in the book "The Hadley Circulation: Present, Past and Future" (Diaz and Bradley, 2004) and a clear explanation of theoretical aspects can be found in Chapter 11 of "Atmospheric and Oceanic Fluid Dynamics: Fundamentals and Large-scale Circulation" (Vallis, 2006). The book "An Introduction to the Dynamics of El Niño and the Southern Oscillation" (Clarke, 2008) will give you a good start on tropical interannual variability. Chapters 6 to 11 of "The global circulation of the atmosphere" (Schneider and Sobel, 2007) address theoretical aspects of the tropical dynamics and its interaction with precipitation. An article by Li et al. (Journal of Climate, 2015) sums up the influence of cloud radiative effects on atmospheric circulation.

\section{Acknowledgments}

The authors are grateful to Dominique Bouniol and Françoise Guichard for inspiring discussions, and to David Saint-Martin for his multiple comments. Thanks to Cathy Hohenegger, Christopher Holloway, and Brian Mapes for their reviews.

\section{Figure credits}

Figure 9.3 courtesy of Norbert Noreiks.

Figure 9.4 courtesy of Stéphanie Leroux.

Figure 9.5 courtesy of Emily Riley.

\section{Exercises}

\section{Damped Gravity Wave approximation}

Derive Equation 9.8.

\section{Walker circulation}

We can build a "simple", linear model of the Walker circulation responding to latent and surface heating. We use the DGW approximation to describe the Walker circulation as a wave extending across the Pacific ocean, with the zonal wavenumber $k=\pi / L_{x}$, with $L_{x}$ the width of the equatorial Pacific. We also neglect the contribution of humidity to the density temperature anomaly, so that $\hat{T}_{\rho}=\hat{T}$. Since damping is larger at the surface than above, we will opportunistically set the damping rate proportional to the pressure: $\eta=\nu p$. We will also consider that the surface and the tropopause are isobaric surfaces at $p_{\mathrm{s}}=1000 \mathrm{hPa}$ and $p_{\mathrm{t}}=150 \mathrm{hPa}$.

Together with Equation (9.8), we use the linearized thermodynamic equation (2.100) at equilibrium, in which we consider that horizontal advection and clear-sky radiation act as a damping on the wave:

$$
\hat{\omega} \Gamma+\hat{Q}_{\mathrm{c}}+\hat{Q}_{\mathrm{r}}+\hat{Q}_{\mathrm{t}}-\mu \hat{T}=0,
$$

in which $\Gamma=-\partial_{p}\left(c_{e} T_{\text {ref }}+\phi_{\text {ref }}\right)>0$ is the opposite of the basic-state vertical gradient of static energy and $\nabla \cdot \boldsymbol{q}$ has been divided into three terms: $\hat{Q}_{\mathrm{r}}$ is the cloud radiative heating, $\hat{Q}_{\mathrm{t}}$ is the turbulent heating, and $\mu$ is the damping rate due to clear-sky radiation and horizontal advection. Equation E.9.1 relates the perturbation to a sum of forcings and, since the model is linear, the total circulation due to the sum of forcings equals the sum of circulations due to each forcing.

a. Walker circulation forced by latent heating: we set $\hat{Q}_{\mathrm{r}}$ and $\hat{Q}_{\mathrm{t}}$ to zero.

i. Establish the relationship between the pressure velocity $\hat{\omega}$ and the latent heating $\hat{Q}_{\mathrm{c}}$. We will use $\alpha=R_{\mathrm{d}} k^{2} / \nu / \mu$ to simplify the expressions.

ii. What are the solutions to the corresponding homogeneous equation? (A change of variable makes it easy.)

iii. Assuming that the heating profile is described by a polynomial $\hat{Q}_{\mathrm{c}}=\sum_{n=0}^{N} \mathcal{Q}_{n} p^{n}$, look for a polynomial particular solution $\hat{\omega}=\sum_{n=0}^{N} \Omega_{n} p^{n}$ to the non-homogeneous equation established in question i.

iv. Using the boundary conditions of no vertical velocity at the tropopause $\left(p=p_{\mathrm{t}}\right)$ and surface $\left(p=p_{\mathrm{s}}\right)$, write the solution to the equation established in question $\mathrm{i}$ for a polynomial heating $\hat{Q_{\mathrm{c}}}$.

v. Let's assume that convective and stratiform clouds produce heating profiles described by the following normalized profiles $\zeta_{\mathrm{c}}(p)$ and $\zeta_{\mathrm{s}}(p)$ :

$$
\zeta_{\mathrm{c}}(p)=6 \frac{\left(p-p_{\mathrm{t}}\right)\left(p_{\mathrm{s}}-p\right)}{\left(p_{\mathrm{s}}-p_{\mathrm{t}}\right)^{3}}
$$

and

$$
\zeta_{\mathrm{s}}(p)=60 \frac{\left(p-p_{\mathrm{t}}\right)\left(p_{\mathrm{m}}-p\right)\left(p_{\mathrm{s}}-p\right)}{\left(p_{\mathrm{s}}-p_{\mathrm{t}}\right)^{4}},
$$

with $p_{\mathrm{m}}=\left(3 p_{\mathrm{s}}+2 p_{\mathrm{t}}\right) / 5=660 \mathrm{hPa}$ the pressure where the stratiform heating changes sign. The amplitude of the vertically integrated latent heating in the WPCZ is about $100 \mathrm{~W} / \mathrm{m}^{2}$ (corresponding to an east-west contrast of $200 \mathrm{~W} / \mathrm{m}^{2}$ or $7 \mathrm{~mm} /$ day). What would be the profile of the pressure velocity if all the precipitation was con- 


\begin{tabular}{|c|c|c|c|c|}
\hline$k$ & $\nu$ & $\mu$ & $\Gamma$ & $\alpha$ \\
\hline $2.5 \cdot 10^{-7} \mathrm{~m}^{-1}$ & $2 \cdot 10^{-10} \mathrm{~s}^{-1} \mathrm{~Pa}^{-1}$ & $5 \cdot 10^{-3} \mathrm{~J} \mathrm{~s}^{-1} \mathrm{~K}^{-1}$ & $0.4 \mathrm{~J} \mathrm{~Pa}^{-1}$ & $18 \mathrm{~kg} \mathrm{~m}^{-3} \mathrm{~s}^{-1}$ \\
\hline
\end{tabular}

Table 9.1. Parameter values.

vective? What if all the precipitation was stratiform? What if half the precipitation is stratiform, as observed in the WPCZ? Plot the profiles of latent heating $\hat{Q_{\mathrm{c}}}$ and the corresponding profiles of $\hat{\omega}, \hat{\phi}$, and $\hat{T}$. What error on the Walker circulation is made if no stratiform precipitation is simulated? Use the constants $R_{\mathrm{d}}=288 \mathrm{~J} \mathrm{~kg}^{-1} \mathrm{~K}^{-1}, g=10$ $\mathrm{m} \mathrm{s}^{-2}$, and the parameter values given in Table 9.1

vi. In the solution found in question iv, we have both the response of the circulation to the latent heating (term in $\hat{Q}_{\mathrm{c}}$ in the original differential equation from question i), and the feedback of the advection (term in $\hat{\omega} \Gamma$ ). We can investigate the role of each contribution. For the realistic case with a stratiform to convective ratio of one, plot the temperature perturbation $\hat{T}_{\mathrm{d}}$ due to the diabatic heating and the perturbation $\hat{T}_{\omega}$ due to the circulation feedback. Derive and plot the corresponding geopotential and pressure velocity perturbations $\hat{\phi}_{\mathrm{d}}, \hat{\phi}_{\omega}, \hat{\omega}_{\mathrm{d}}$, and $\hat{\omega}_{\omega}$ (a change of variable might help again). What is the effect of the circulation feedback on the dynamics? What would be its effect on precipitation if precipitation was interactive?

b. Walker circulation forced by the SST gradient: we set $\hat{Q}_{\mathrm{r}}$ and $\hat{Q}_{\mathrm{c}}$ to zero and we assume that $\hat{Q}_{\mathrm{t}}$ communicates the SST gradient very efficiently up to the top of the boundary layer at $p_{\mathrm{i}}=800 \mathrm{hPa}$, so that the air temperature anomaly is equal to the SST anomaly $\hat{T}_{\mathrm{S}}$ up to $p_{\mathrm{i}}: \hat{T}=\hat{T}_{\mathrm{S}}$ for $p>p_{\mathrm{i}}$.

i. Establish the differential equations for $\hat{\omega}$ below and above $p_{\mathrm{i}}$.

ii. Solve for $\hat{\omega}$ using the boundary conditions of zero vertical velocity at the surface and tropopause, as well as the continuity of $\hat{\omega}$ and $\partial_{p} \hat{\omega}$ at $p_{\mathrm{i}}$ required by mass conservation and the continuity of the geopotential.

iii. Plot the profiles of $\hat{\omega}, \hat{\phi}$, and $\hat{T}$ for a perturbation $\hat{T}_{\mathrm{S}}=5 \mathrm{~K}$ (corresponding to an east-west SST contrast of $10 \mathrm{~K})$.

iv. Plot the profiles of the perturbations of vertical circulation, temperature, and geopotential due to the "realistic" latent heating and the SST gradient. What is the contribution of each term?

\section{c. Walker circulation forced by cloud radiative effect:}

On the basis of the cloud radiative effects depicted in
Figure 9.11, what contribution to the Walker circulation do you expect from the clouds in the WPCZ?

\section{Horizontal circulation: effect of rotation and $\beta$ ef- fect}

This simple exercise aims at illustrating how the dynamical response of the tropical atmospheric circulation to a geopotential perturbation does not conserve the symmetries of the geopotential field, which can be instrumental in the propagation of the disturbance. We use the linearized primitive equation for momentum (9.1) with a motionless basic state and a damping term that account for vertical diffusion and surface friction, that we rewrite for the zonal and meridional winds $u$ and $v$ :

$\partial_{t} u-f v=-\partial_{x} \phi-\eta u$,

$\partial_{t} v+f u=-\partial_{y} \phi-\eta v$,

We will use $\eta=210^{-5} \mathrm{~s}^{-1}$ typical of the atmospheric boundary layer.

a. Establish the equation for the steady horizontal wind $(u, v)$ and divergence $\delta=\partial_{x} u+\partial_{y} v$ for a steady geopotential perturbation.

b. Let's consider a Gaussian geopotential perturbation, that could result from cloud diabatic heating above the level under consideration:

$$
\phi=-\phi_{0} \exp \left(-\frac{x^{2}+y^{2}}{2 L^{2}}\right)
$$

with $\phi_{0}=500 \mathrm{~m}^{2} \mathrm{~s}^{-2}$ the perturbation amplitude and $L=100 \mathrm{~km}$ the horizontal scale of the perturbation. Plot the wind and divergence response in the case without rotation, in the case of the equatorial $\beta$ plane $\left(f=\beta y\right.$ with $\left.\beta=2.2710^{-11} \mathrm{~m}^{-1} \mathrm{~s}^{-1}\right)$, for a subtropical $f$-plane $\left(f=f_{0}=1.2710^{-5} \mathrm{~s}^{-1}\right)$ around $15^{\circ} \mathrm{N}$, and for the corresponding $\beta$-plane $\left(f=f_{0}+\beta y\right.$ with $f_{0}=1.2710^{-5} \mathrm{~s}^{-1}$ and $\beta=2.2010^{-11} \mathrm{~m}^{-1} \mathrm{~s}^{-1}$ ). Does the divergence field conserve the symmetry of the geopotential field in the case without rotation? On an $f$-plane? On a $\beta$-plane?

c. How does the circulation feed back on such a geopotential perturbation in the boundary layer? What can we expect from the energy advection and the moisture advection?

\section{Gill circulation}

The damped, baroclinic dynamical response to a localized, steady, mid-tropospheric heating is called Gill circulation. To describe this circulation, we can use the primitive equations for continuity 9.2 , hydrostatic $(9.3)$, and momentum 9.1 on the equatorial $\beta$-plane with a damping term, together with the thermodynamic equa- 
tion (2.100) linearized about a motionless basic state. We also neglect the influence of the humidity on the density temperature, so that $T_{\rho}=T$, and we approximate $c_{e}$ by $c_{p, \mathrm{~d}}$. With these simplifications, the equations are:

$$
\begin{gathered}
\partial_{t} \boldsymbol{v}_{p}+\beta y \mathbf{k} \times \boldsymbol{v}_{p}=-\nabla_{p} \phi-\eta \boldsymbol{v}_{p}, \\
\nabla_{p} \cdot \boldsymbol{v}_{p}+\partial_{p} \omega=0, \\
\partial_{p} \phi=-\frac{R_{\mathrm{d}} T}{p} \\
c_{p, \mathrm{~d}} \partial_{t} T-\omega \Gamma=Q-\eta c_{p, \mathrm{~d}} T,
\end{gathered}
$$

with $\eta$ the damping rate, $\Gamma=-\partial_{p}\left(c_{e} T_{\text {ref }}+\phi_{\text {ref }}\right)>0$ the opposite of the basic-state vertical gradient of static energy, $Q=\nabla \cdot \boldsymbol{q}+Q_{\mathrm{c}}$ the prescribed heating, and $\mathbf{k}$ the unit vertical vector. The damping coefficient $\eta$ is chosen to be the same for momentum and energy to simplify the mathematics. We will also consider that the surface and the tropopause are isobaric surfaces at $p_{\mathrm{s}}=1000 \mathrm{hPa}$ and $p_{\mathrm{t}}=150 \mathrm{hPa}$.

We prescribe the vertical profile of (positive) heating $\mathcal{Q}(p)$ with a maximum in the middle troposphere, so that $Q=\mathcal{Q}(p) Q_{1}$. We choose a normalized profile:

$\langle\mathcal{Q}\rangle=1 \quad$ with $\quad<\cdot>=\int_{p_{\mathrm{t}}}^{p_{\mathrm{s}}} \cdot d p$.

We also assume that the profile of temperature anomaly follows the heating profile: $T=\mathcal{Q}(p) T_{1}$, and we set consistent profiles for the baroclinic wind $\mathcal{V}(p)$ and the associated vertical wind $\Omega(p)$ so that $\boldsymbol{v}_{p}=\mathcal{V}(p) \boldsymbol{v}_{1}$ and $\omega=\Omega(p) \omega_{1}$.

a. Normalized equations of the baroclinic wind

i. Use the hydrostatic and momentum equations to express $\mathcal{V}(p)$ as a function of $\mathcal{Q}(p)$, and use the equation of continuity to express $\Omega(p)$ as a function of $\mathcal{V}(p)$ (traditionally, the baroclinic wind profile is set positive in the upper troposphere and negative in the lower troposphere).

ii. Establish the equation of the baroclinic wind $\boldsymbol{v}_{1}$ from the momentum and hydrostatic equations; establish the equation for the temperature perturbation $T_{1}$ using the continuity equation and the vertically integrated thermodynamic equation. What is the difference between this system and the shallow-water equations? How can we express the gravity-wave speed $c$ ?

iii. Non-dimensionalize the equations using the equatorial Rossby radius $(c / 2 \beta)^{1 / 2}$ (about $10^{\circ}$ of latitude) and the time scale $(2 \beta c)^{1 / 2}$ (about 6 hours).

\section{b. Method of solution}

We further assume that the damping term and tendency of the meridional wind $v$ is negligible compared to the others and rewrite the equations with the nondimensional variables $\hat{q}=\hat{T}+\hat{u}$ and $\hat{r}=\hat{T}-\hat{u}$, with $u$ the zonal wind and $\hat{\imath}$ indicating the non-dimensional variable.

i. Omitting the hats, establish the following equations for the non-dimensional variables $q, r$, and $v$, the non-dimensional heating $Q$ and damping rate $\eta$ :

$$
\begin{aligned}
\eta q+\partial_{t} q+\partial_{x} q-\frac{1}{2} y v+\partial_{y} v & =Q, \\
\eta r+\partial_{t} r-\partial_{x} r+\frac{1}{2} y v+\partial_{y} v & =Q, \\
\partial_{y} q+\frac{1}{2} y q+\partial_{y} r-\frac{1}{2} y r & =0
\end{aligned}
$$

ii. Reduce this set of equations to a single equation in $v$.

iii. Using the separation of variables $v=$ $A(t) B(x) D(y)$, show that the solutions to the corresponding homogeneous equation (with $Q=0$ ) have parabolic cylinder meridional structures $D_{n}(y)=P_{n}(y) \exp \left(-y^{2} / 4\right)$, with $P_{n}$ a degree- $n$ polynomial. Give the expression of $P_{0}, P_{1}$, and $P_{2}$. Establishing two iterative relationships for $D_{n}$, show that the meridional structures of $q$ and $r$ are also these parabolic functions.

iv. We look for solutions of the forced problem by expanding the forcing $Q$ and variables $q, r$, and $v$ in terms of these parabolic cylindric functions $D_{n}$ :

$$
Q=\sum_{n=0}^{\infty} Q_{n}(x, t) D_{n}(y), \text { etc. }
$$

Establish the equation system for the variables $q_{n}, r_{n}, v_{n}$.

\section{c. Steady forcing}

The Gill circulation per se is the response to a steady heating. Here we will limit our study to the case of a simple heating symmetric about the equator:

$$
\begin{array}{lll}
Q_{0}=\cos k x & \text { for }-L \leq x \leq L, \\
Q_{0}=\quad 0 & \text { for }|x|>L, \\
Q_{n}=\quad 0 & \text { for } n>0,
\end{array}
$$

with $k$ the zonal wavenumber and $L=\pi / 2 / k=2$. We look for the equilibrium response, so that $\partial_{t}=0$.

i. Show that all the $q_{n}, r_{n}$, and $v_{n}$ are zero except $q_{0}, r_{0}, v_{1}$, and $q_{2}$.

ii. What is the solution $q_{0}^{0}$ to the homogeneous equation? Look for a particular solution in $\sin k x$ and $\cos k x$ for $-L \leq x \leq L$ and find the solution for $q_{0}$.

iii. What is the solution $r_{0}^{0}$ to the homogeneous equation? Look for a particular solution for $-L \leq x \leq L$ and find the solution for $r_{0}$. Deduce the expressions of $q_{2}$ and $v_{1}$.

iv. Plot the surface winds, convergence, and temperature response to the steady heating for a damping rate $\eta=0.1$. 
v. Why is the temperature perturbation not collocated with the heating? What can we expect from moist feedbacks if humidity is taken into account?

\section{d. Propagating forcing}

We now consider a propagating heating with a phase speed $c$ that we choose eastward to represent the MJO:

$Q_{0}=\cos [k(x-c t)] \quad$ for $c t-L \leq x \leq c t+L$,

$Q_{0}=\quad 0 \quad$ for $x<c t-L$ or $x>c t+L$,

$Q_{n}=\quad 0 \quad$ for $n>0$.

We are looking for a propagating solution $(q, r, v)$ functions of $x^{\prime}=x-c t$, so that $\partial_{x}=\partial_{x^{\prime}}$ and $\partial_{t}=-c \partial_{x^{\prime}}$

i. Rewrite the equations for $q_{n}, r_{n}$, and $v_{n}$ using $x^{\prime}$ as variable.

ii. Deduce which $q_{n}, r_{n}$, and $v_{n}$ are non-zero. Give the solution for $q_{0}$.

iii. Find the solutions for $r_{0}, q_{2}$, and $v_{1}$.

iv. Plot the surface winds, convergence, and temperature response to the propagating heating, for $t=0\left(x^{\prime}=x\right)$, for a non-dimensional phase speed $c=0.1$ (corresponding to a dimensional phase speed of about $5 \mathrm{~m} \mathrm{~s}^{-1}$ ), and the difference with the response to steady heating. Should we expect the MJO to exhibit a dynamical pattern similar to the equilibrium dynamical response to the diabatic heating? 